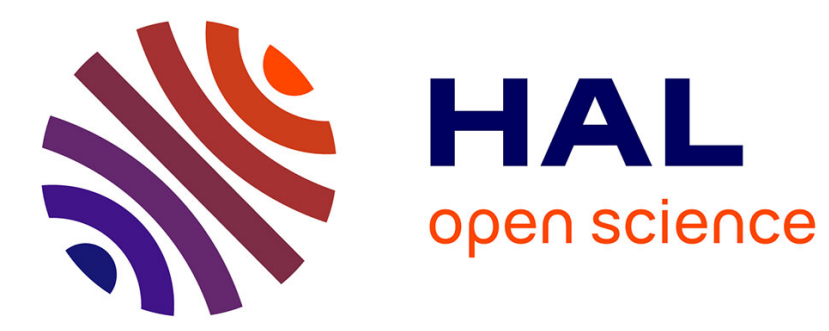

\title{
Modelling net carrying amount of shares for market consistent valuation of life insurance liabilities
}

Diana Dorobantu, Yahia Salhi, Pierre-Emmanuel Thérond

\section{To cite this version:}

Diana Dorobantu, Yahia Salhi, Pierre-Emmanuel Thérond. Modelling net carrying amount of shares for market consistent valuation of life insurance liabilities. Methodology and Computing in Applied Probability, 2020, 22, pp.711-745. hal-01840057

\section{HAL Id: hal-01840057 https://hal.science/hal-01840057}

Submitted on 16 Jul 2018

HAL is a multi-disciplinary open access archive for the deposit and dissemination of scientific research documents, whether they are published or not. The documents may come from teaching and research institutions in France or abroad, or from public or private research centers.
L'archive ouverte pluridisciplinaire HAL, est destinée au dépôt et à la diffusion de documents scientifiques de niveau recherche, publiés ou non, émanant des établissements d'enseignement et de recherche français ou étrangers, des laboratoires publics ou privés. 


\title{
Modelling net carrying amount of shares for market consistent valuation of life insurance liabilities*
}

\author{
Diana Dorobantu, ${ }^{(1)}$ Yahia SAlhi ${ }^{(1)}$ and Pierre-E. Thérond ${ }^{(1,2)}$ \\ (1) ISFA, Univ Lyon, UCBL, LSAF EA2429, F-69007, Lyon, France \\ ${ }^{(2)}$ Galea \& Associés, 25 rue de Choiseul, F-75002, Paris, France
}

July 16, 2018

\begin{abstract}
The attractiveness of insurance saving products is driven, among others, by dividends payments to policyholders and participation in profits. These are mainly constrained by regulatory measures on profit-sharing on the basis of statutory accounts. Moreover, since both prudential and financial reporting regulation require market consistent best estimate measurement of insurance liabilities, cash-flows projection models have to be used for such a purpose in order to derive the underlying financial incomes. Such models are based on Monte-Carlo techniques. The latter should simulate future accounting profit and losses needed for profit-sharing mechanisms.

In this paper we deal with impairment losses on equity securities for financial portfolios which rely on instrument-by-instrument assessment (when projection models consider groups of shares). Our motivation is to describe the joint distribution of market value and impairment provision of a book of equity securities, with regard to the French accounting rules for depreciation. The results we obtain enable to improve the ability of projection models to represent such an asymmetric mechanism. Formally, an impairment loss is recognized for an equity instrument if there has been a significant and prolonged decline in its market value below the carrying cost (acquisition value). Such constraints are formalized using an assumption on the dynamics of the equity, and leads to a complex option-like pay-off.

Using this formulation, we propose analytical formulas for some quantitative measurements related the impairments losses of a book of financial equities. These are derived on a general framework and some tractable example are illustrated. We also investigate the operational implementation of these formulas and compare their computational time to a basic simulation approach.
\end{abstract}

Keywords: Insurance, Best Estimate Technical Provision, Impairment Losses, Correlated Brownian Motions, Joint Density

*This work has been supported by the BNP Paribas Cardif Chair "Data Analitycs and Models in Insurance". The views expressed in this document are the authors owns and do not necessarily reflect those endorsed by BNP Paribas Cardif. Contacts: D. Dorobantu (diana.dorobantu@univ-lyon1.fr), Y. Salhi (yahia.salhi@univ-lyon1.fr) and P.-E. Thérond (pierre@therond.fr). 


\section{Introduction}

Over the past decade, market consistent best estimate measurement became the new standard when it comes to life insurance valuation. This principle was first generalized by the Market Consistent Embedded Value (MCEV) principles published by the CFO Forum in 2009 and was then considered by the European Union for prudential purposes (Solvency II) and by the International Accounting Standard-Setter (IASB) for financial reporting purposes, see Thérond (2016), Vedani et al. (2017) and Wüthrich (2016).

Due to the complexity of life insurance mechanisms, in most cases, this kind of measurement requires to use some cash-flows projection models in order to estimate the market consistent best estimate using Monte Carlo simulations. As a matter of fact, projecting cash-flows of life insurers requires modelling future guarantees revaluation and policyholders behaviours (especially regarding lapses) which both are dependent on future economic scenarios. These economic scenarios have to be drawn consistently with the current observed prices on the market (see Thérond (2016) for more details on market consistency principles). In order to comply with the market consistency, risk neutral approaches appear to be the present standard even if some other approaches would meet the requirement (e.g. real world economic scenarios coupled with stochastic deflators when it comes to discount future cash-flows). Each generated scenario should take into account the behaviours of insurer (through asset management, revaluation policy, etc.) but also of policyholders (mortality, partial or total lapses, etc.).

Moreover, in some jurisdictions (e.g. Italy, Germany or France), participation mechanisms are constrained by regulation. For example, in France, life insurers have to dedicate, at least, $85 \%$ of financial incomes to re-evaluate policyholder's annuities or savings. Here, financial incomes has to be understood as local Generally Accepted Accounting Principles (GAAP) financial revenues. As a consequence, and in order to apprehend this kind of constraints, local GAAP financial statements have to be projected, too.

Modelling local GAAP financial statements appears to be quite a challenge due to the complexity of some accounting mechanisms and the granularity at which they have to be considered. It is especially the case for projecting financial assets due to the impairment provisions which are recognized on an instrument-by-instrument basis and may have a significant impact on local GAAP financial revenue and thus on the constraints an insurer has to deal with when considering revaluation decisions.

As a matter of fact, life insurers have to deal with guaranteed rate and participation features. Cashflows projection models have to be able to incorporate management rules such as the asset-and-liability management (ALM), e.g. asset management policy regarding the entity's objectives (giving good bonuses to policyholders in order to avoid lapses, keeping some unrealized gains to prevent the consequences of potential future market drops, containing P\&L's volatility, etc.) Equity securities management play a major role in this ALM, since selling shares with unrealized gains and losses enables to modify the local GAAP financial result. As a consequence, it is of major importance to be able to precisely model the correlated behaviour of the market value and net carrying amounts of such financial assets.

Such a problem is the main motivation of this work. In fact, our main goal is to derive some analytical formulas in order to be able to simulate the joint carrying amount and market value of financial equity portfolios, regarding French impairment rules. This subject is of paramount importance, from an operational perspective, since cash-flows projecting models generally do not consider individual financial assets but groups of financial assets (model points). The main challenge consists in deriving this joint distribution for a book of $n$ assets without computing the impairment mechanism for each individual asset.

In this paper, we propose closed form formulas of impairment provisions for a book of $n$ equities with different assumptions on their dependency. First, we consider the case of comonotonic shares driven by the same Brownian motion. In fact, the valuation principle associated with the acquisition cost measurement leads to distinctly recognize several lines of the same title acquired at different times, thus at different 
costs. So we may have, if any, different levels of impairment reserves for the same security but acquired at different dates. Recently Azzaz et al. (2015) considered a similar case but with a different impairment rule. Indeed, they investigated a criteria based on a separated events, e.g. decline and prolonged events. However, in that paper the authors only considered a single equity. Therefore, in the current work we intend to extend the results of Azzaz et al. (2015) to a more general framework but with a joint realisation of the above mentioned impairment conditions. We should note that one can also derive the quantities of interest in the framework of Azzaz et al. (2015) using the result developed in Section 4, e.g. next-period expected impairment and its cumulative distribution function (c.d.f.). Secondly, we are tackling the case of linearly dependent shares. In a similar way, we are able to develop analytical of the distributional specificities of next-period impairment. This is mainly based on the existence of the joint law of a multidimensional drifted Brownian motion with a vector of its element-wise supremum over a given period. We should note that in our case we did use the joint density to characterize the quantities of interest. This density is known for $n=2$ but its analytical form for $n \geq 3$ does only exist for some particular correlation matrices, see Escobar and Hernandez (2014). We thus propose the computation of the next period impairment c.d.f. of some particular cases where the aforementioned density does exist based on the method of images, see Keller (1953).

Finally, we derive the explicit form of the joint c.d.f. of the aggregate carrying amounts and the impairment losses both for the comonotonic and linearly dependent shares. This c.d.f. is of paramount importance in practice as it should help the determination of a representative share of a book of equities when a model point approach is considered in the internal model of the insurer. In fact, due to constraints on computation times and implementation costs but also due to the complexity of a share-by-share approach, insurers generally rely on a model point that represents a book of equity, see Bennemann and Hennig (2010). This leads to the application of the accounting depreciation rules to the representative equity instead of applying it individually to each security. The results obtained in Azzaz et al. (2015) (with a slightly different impairment criterion, that of IAS 39) have shown the inadequacy of the representation of impairments using the only significant loss criterion. Hence, using this joint c.d.f. should enhance the construction of the model point.

The remainder is organized as follows. In Section 2 we introduce the accounting standards that French insurers have to apply for accounting non-depreciable financial assets (e.g. funds and stocks). This gives arise to a mathematical formulation for the impairment rule as a complex option-like cash-flow. In Section 3, we formulate this problem using a Black and Scholes framework, where the equities follow a geometric Brownian motion. The impairment is formulated and its distribution and expectation are investigated for a single share and give the basis for the computations for a book of $n$ equities that follow in Section 4. In this section, we introduce the main result of the paper while distinguishing comonotonic of linearly dependent shares. Finally, in Section 5 we investigate the computational performance of the analytical results and analyse the bias estimation stemming from a basic simulation-based approach.

\section{Accounting measurement of equity securities}

2.1 General Measurement. In France, the revaluation of life insurance policies is constrained by regulatory measures on profit-sharing on the basis of statutory accounts. Therefore, it is necessary to describe the specific modalities that apply to the measurement and recognition of the financial assets. Precisely, the accounting standards for equity instruments are governed by Art. R343-10 of the French Insurance Code and sections 123-1 to 123-19 of the French Insurance Accounting Regulation ${ }^{1}$ for their

\footnotetext{
${ }^{1}$ Règlement ANC n ${ }^{\circ} 2015-11$ available at https://www.anc.gouv.fr
} 
depreciation methods ${ }^{2}$. On an individual basis, these rules can be summarized as follows:

(i) at initial recognition, an equity security is measured and recognized at its acquisition cost;

(ii) the subsequent measurement of that kind of financial instruments remains at their acquisition cost;

(iii) under some conditions (described below), an impairment provision may be recognized.

From this accounting scheme, at each annual reporting date, the net carrying amount of an equity security is equal to its acquisition cost less the impairment provision. The differences between the opening and closing values of the impairment provision are recognized through profits-and-losses (P\&L). When this equity security is sold during the reporting period, the difference between its net carrying amount (at the opening) and the sold price is recognized through P\&L.

2.2 Impairment losses and provision. French accounting regulations state that provisions for impairment have to be accounted on an individual basis, i.e. unrealised gains from some shares do not compensate unrealised losses from other for the purpose of determining such provisions. Considering an individual financial asset, an impairment provision has to be recognized if, at reporting date:

(i) the market value is inferior to the acquisition value; and

(ii) the unrealized loss presents a durable nature.

For non-amortizable financial assets, several cases have to be considered regarding the durable or permanent condition. So, an unrealized loss of an equity security is presumed durable if:

(i) an impairment provision was recognized in the opening balance sheet;

(ii) it exists some objective hints that the unrealized loss presents a durable nature;

(iii) the unrealized loss has been significant and prolonged.

In the following, we will focus on the last criterion which is a quantitative one. Unlike the IAS 39 standards (see Azzaz et al. (2015)), the French standard defines how to consider a significant and prolonged impairment : when the unrealized loss was higher than $20 \%$ of the acquisition cost during the latest 6-months. Beyond the impairment trigger, two other disposals are quite different from IAS 39 impairment mechanism:

(i) the impairment provision is re-evaluated each year (with the possibility of reversal),

(ii) if the insurance company has the will and the ability to hold the equity security over the next years, the impairment provision could be lesser than the unrealized loss ${ }^{3}$.

In the following, without loss of generality, we will not consider this latter possibility.

2.3 Notation. In this section, we consider the impairment of a single asset $i$, denoted $S^{i}$, according to the accounting principles discussed above. Suppose that we are at the beginning of the period $t$ and we seek to quantify the $(t+1)$-period impairment. At the end of this period, the equity is impaired whenever its recoverable amount falls under the acquisition value $S_{t_{i}}^{i}=a_{i}>0$ ( $t_{i}$ denotes the acquisition date). This rule must be considered jointly with the constraint on the prolonged effect of the drop-down. Indeed, the impairment is triggered when the observed market value over the period $[t+1 / 2, t+1]$ remained below a given level $\alpha$ of the acquisition value, where $\alpha \in[0,1]$. These together induce a recognition of a loss $\left(a_{i}-S_{t+1}^{i}\right)$. More formally, given the information acquired up to time $t>t_{i}$ and denoting $\widetilde{S}_{t+1}^{i}=\sup _{u \in[t+1 / 2, t+1]} S_{u}^{i}$, the future impairment $\mathrm{PDD}_{t+1}^{i}$ is given as follows:

$$
\mathrm{PDD}_{t+1}^{i}=\left(a_{i}-S_{t+1}^{i}\right)^{+} \mathbb{1}_{\widetilde{S}_{t+1}^{i} \leq \alpha a_{i}}
$$

\footnotetext{
${ }^{2}$ The details on the French insurance regulation is available at https://www.legifrance.gouv.fr

${ }^{3}$ The insurance company may compute a so-called "recoverable value" corresponding to the market value projected over the remaining expected holding period (with a maximum of 5 years) of the considered financial asset.
} 
As a consequence, the net accounting value of this title is given by

$$
\mathrm{NAV}_{t+1}^{i}=a_{i}-P D D_{t+1}^{i}
$$

\section{Model assumptions}

The issue we want to deal with consists in the projection of both net accounting and market values of equity securities belonging to a life insurer's financial assets portfolio.

As mentioned previously, both new prudential and financial reporting standards require market consistent valuation of insurance liabilities. Concerning with-profits life insurance contracts, in most case, such evaluations are conducted using Monte Carlo simulations based on future cash-flows realisations drawn from a projection model. Such a model has to represent asset-and-liability management, especially local GAAP P\&L consequences of selling (or not) financial assets. Moreover, since Solvency II and IFRS 17 require market consistent measurement, in most cases, risk-neutral economic scenario generators are considered. More specifically, the stock dynamics are evolving under the risk neutral probability measure. This practice roots in the use of the quantified provisions to assess the market consistent of the liability best estimate under the regulatory requirement. As noted before, the latter should take into account the financial returns that are accounted for in a profit sharing mechanism.

Accordingly, under the risk neutral measure, the equities share have the same drift rate that is equal to the risk-free rate but have different volatilities. Hereafter, we consider that the dynamics of these stock prices can be described by a geometric Brownian motion as follows

$$
d S_{t}^{i}=S_{t}^{i}\left(r d t+\sigma_{i} d W_{t}^{i}\right), \quad t \geq 0
$$

where $r$ and $\sigma^{i}$ are some constant parameters referring, respectively, to the risk-free interest rate and the volatility of the $i^{\text {th }}$ equity. Here, $W^{i}=\left(W_{t}^{i}\right)_{t \geq 0}$ is a standard Brownian motion such that for each $i \neq j$ we have $\operatorname{cov}\left(W_{t}^{i}, W_{t}^{j}\right)=\rho_{i j} t$, where $\left.\rho_{i j} \in\right]-1,1[$ is the correlation coefficient. Moreover, let us introduce the following notation

$$
X_{t}^{i}=\mu_{i} t+\sigma_{i} W_{t}^{i}, \quad \text { where } \mu_{i}=\frac{2 r-\sigma_{i}^{2}}{2},
$$

which will be used throughout the sequel.

A primal quantity of interest when dealing with impairment is the distributional behaviour of the impairment provision at a given time. To this end, the next proposition gives the c.d.f. of the impairment provision of a single title at time $t$, given the available information at time $s$. In the following proposition, the notation $\mathbb{P}_{t}(\cdot)$ (resp. $\mathbb{E}_{t}(\cdot)$ ) stands for the conditional probability $\mathbb{P}\left(\cdot \mid \mathcal{F}_{t}^{i}\right)$ (resp. conditional expectation $\mathbb{E}\left(\cdot \mid \mathcal{F}_{t}^{i}\right)$ ) where $\mathcal{F}_{t}^{i}$ is the filtration generated by the Brownian motion $W^{i}$, i.e. $\mathcal{F}_{t}^{i}=\sigma\left\{W_{s}^{i}, s \leq t\right\}$.

Proposition 1 Consider an asset $i$ with acquisition value $a_{i}>0$, acquisition date $t_{i}$ and future impairment $P D D^{i}$ given by Equation (2.2). Let $t>\frac{1}{2}$. For all $t_{i} \leq s<t-\frac{1}{2}$, the cumulative distribution function of $P D D_{t}^{i}$ is given as follows:

$$
\mathbb{P}_{s}\left(P D D_{t}^{i} \leq x\right)=\left\{\begin{array}{ccr}
0 & \text { if } & x<0, \\
p_{1}\left(a_{i}, S_{s}^{i}\right) & \text { if } & 0 \leq x<a_{i}(1-\alpha), \\
p_{2}\left(a_{i}, S_{s}^{i}\right) & \text { if } & a_{i}(1-\alpha) \leq x<a_{i}, \\
1 & \text { if } & x \geq a_{i},
\end{array}\right.
$$


with

$p_{1}\left(a_{i}, S_{s}^{i}\right)=1-\Phi\left(w, \frac{\ln \left(\frac{\alpha a_{i}}{S_{s}^{i}}\right)-\mu_{i} \delta}{\sigma_{i} \sqrt{\delta}} ; \frac{\sqrt{2 \delta-1}}{\sqrt{2 \delta}}\right)+\left(\frac{\alpha a_{i}}{S_{s}^{i}}\right)^{\frac{2 \mu_{i}}{\sigma_{i}^{2}}} \Phi\left(w+\frac{2 \mu_{i}}{\sigma_{i}} \sqrt{\delta-\frac{1}{2}}, \frac{-\ln \left(\frac{\alpha a_{i}}{S_{s}^{i}}\right)-\delta \mu_{i}}{\sigma_{i} \sqrt{\delta}} ;-\frac{\sqrt{2 \delta-1}}{\sqrt{2 \delta}}\right)$,

$p_{2}\left(a, S_{s}^{i}\right)=1-\Phi\left(w, \frac{\ln \left(\frac{a_{i}-x}{S_{s}^{i}}\right)-\mu_{i} \delta}{\sigma_{i} \sqrt{\delta}} ; \frac{\sqrt{2 \delta-1}}{\sqrt{2 \delta}}\right)+\left(\frac{\alpha a_{i}}{S_{s}^{i}}\right)^{\frac{2 \mu_{i}}{\sigma_{i}^{2}}} \Phi\left(w+\frac{2 \mu_{i}}{\sigma_{i}} \sqrt{\delta-\frac{1}{2}}, \frac{-\ln \left(\frac{\left(a_{i}-x\right) S_{s}^{i}}{a_{i}^{2} \alpha^{2}}\right)-\delta \mu_{i}}{\sigma_{i} \sqrt{\delta}} ;-\frac{\sqrt{2 \delta-1}}{\sqrt{2 \delta}}\right)$,

$w=\left(\ln \left(\frac{\alpha a_{i}}{S_{s}^{i}}\right)-\mu_{i}\left(\delta-\frac{1}{2}\right)\right) / \sigma_{i} \sqrt{\delta-\frac{1}{2}}, \delta=t-s$ and $\Phi(U, V ; \theta)$ is the joint c.d.f. of two correlated Gaussian r.v.s $U$ and $V$ with a given correlation coefficient $\theta$.

Proof. See Appendix A.1.

If this first result enables to simulate future impairment losses, from an operational perspective, we still have to deal with two issues:

(i) the joint evolution of market value and net accounting value has to be considered for applying management rules in projection models;

(ii) insurer's internal models do not consider equity securities on an individual basis (even if the impairment rules apply at this level of measurement).

In the following, we deal with these two issues with the objective of obtaining mathematical results which enable an operational implementation (see 5).

\section{Main results}

In this section, we derive the main quantities needed to assess the impairment of a book of $n$ equities. These are related to the joint distribution of the next period impaired value and the underlying shares values. First, we start with a particular case on which the equities have the same source of uncertainty. This is to take into account a common practice in real world applications where the same Brownian motion is used to generate the dynamics of the stocks and thus compute the related impaired values. This model is also of interest when the insurance company holds many shares of a stock that have been purchased at different dates. Following the accounting rules this share should be split in different lines based on the acquisition date. Secondly, we consider a more general framework where the equity securities are not perfectly correlated. For both frameworks, we denote

$$
\Gamma_{t}=\mathrm{PDD}_{t}^{1}+\cdots+\mathrm{PDD}_{t}^{n}
$$

where $\mathrm{PDD}_{t}^{i}$, for $i=1, \cdots, n$ are defined in Equation (2.2). Moreover, we are also considering the aggregate dynamics $\Sigma=\left(\Sigma_{t}\right)_{t \geq 0}$ defined as follows:

$$
\Sigma_{t}=S_{t}^{1}+\cdots+S_{t}^{n}
$$

From an operational perspective, our main goal is to be able to simulate some realizations of $\left(\Gamma_{t+1}, \Sigma_{t+1}\right)$ conditionally to $\left(\Gamma_{t}, \Sigma_{t}\right)$.

4.1 Portfolio of comonotonic securities. Our first results consist in deriving the joint distribution of the market value and the impairment reserve of shares of the same company, acquired at different times. As a matter of fact, the valuation principle associated with the acquisition cost measurement leads to distinctly recognize several lines of the same title acquired at different times, thus at different costs. So 
we may have, if any, different levels of impairment reserves for the same security but acquired at different dates.

As noted before, in practice, the use of Monte-Carlo simulations based on an asset-by-asset approach often leads to large running times. Therefore, the practitioners are generally used to group the equities into homogeneous groups and to replace the group of contracts with a representative security called a model point, see Bennemann and Hennig (2010). When doing this, they advocate the use comonotonic dynamics for modelling the various securities within each model point. More formally, we consider that the stocks have the same dynamic

$$
d S_{t}=S_{t}\left(r d t+\sigma d W_{t}\right)
$$

where $r, \sigma>0$ and $W=\left(W_{t}\right)_{t \geq 0}$ is a standard Brownian motion. Here $X_{t}=\mu t+\sigma W_{t}$ where $\mu=$ $\left(2 r-\sigma^{2}\right) / 2$. In the other hand, this case could correspond, for instance, to the presence of the same share in the asset book of the insurer with different acquisition dates and values. These are treated separately as the impairment provision does depend on the acquisition value, see Equation (2.2). Given this particular case, our aim is then to derive the joint probability $\mathbb{P}_{t}\left(\Gamma_{t+1} \leq y, \Sigma_{t+1} \leq x\right)$, where as previously mentioned the notation $\mathbb{P}_{t}(\cdot)$ (resp. $\mathbb{E}_{t}(\cdot)$ ) stands for the conditional probability $\mathbb{P}\left(\cdot \mid \mathcal{F}_{t}\right)$ (resp. conditional expectation $\left.\mathbb{E}\left(\cdot \mid \mathcal{F}_{t}\right)\right)$ where $\mathcal{F}_{t}$ is in this section the filtration generated by the Brownian motion $W$, i.e. $\mathcal{F}_{t}=\sigma\left\{W_{s}, s \leq t\right\}$. First, we generalize the result in Proposition 1 and derive a closed form for c.d.f. of the aggregate loss $\Gamma$ defined in Equation (4.1).

Proposition 2 Let consider a book of $n$ shares with different acquisition dates $t_{i}$ and acquisition costs $a_{i}>0, i=1, \ldots, n$. Let $t>\frac{1}{2}$. For all $\max \left(t_{1} \ldots t_{n}\right) \leq s<t-\frac{1}{2}$, the next period probability of recording an impairment on this book is given by

$$
\mathbb{P}_{s}\left(\Gamma_{t}>0\right)=1-p_{1}\left(\max \left(a_{1} \ldots a_{n}\right), S_{s}\right),
$$

where $p_{1}$ is given in Proposition 1.

Proof. See Appendix A.2

Another indicator of interest is the expected loss at the end of a given period. Of course, one can use the c.d.f. in the last proposition to retrieve the expected value of impairments by integration of the survival function. However, this would be painful, and numerically complex. Therefore, in the next result we give the explicit form of the latter conditional on the information up to time $s$.

Proposition 3 For $n$ shares with different acquisition values $a_{i}$ and different acquisition dates $t_{i} \leq s<$ $t-\frac{1}{2}, i=1, \ldots, n$ and $t>\frac{1}{2}$, the next period expected impaired amount is given as follows:

$$
\begin{aligned}
\mathbb{E}_{s}\left(\Gamma_{t}\right) & =\sum_{i=1}^{n} a_{i}\left(1-p_{1}\left(a_{i}, S_{s}\right)\right) \\
& +\sum_{i=1}^{n} e^{r \delta}\left[-S_{s} \Phi\left(\frac{\ln \frac{a_{i} \alpha}{S_{s}}-\left(\mu+\sigma^{2}\right)\left(\delta-\frac{1}{2}\right)}{\sigma \sqrt{\delta-\frac{1}{2}}}, \frac{\ln \frac{a_{i} \alpha}{S_{s}}-\left(\mu+\sigma^{2}\right) \delta}{\sigma \sqrt{\delta}} ; \frac{\sqrt{2 \delta-1}}{\sqrt{2 \delta}}\right)\right. \\
& \left.+\left(a_{i} \alpha\right)^{\frac{2\left(\mu+\sigma^{2}\right)}{\sigma^{2}}} S_{s}^{-\frac{2 r}{\sigma^{2}}} \Phi\left(\frac{\ln \frac{a_{i} \alpha}{S_{s}}+\left(\mu+\sigma^{2}\right)\left(\delta-\frac{1}{2}\right)}{\sigma \sqrt{\delta-\frac{1}{2}}}, \frac{-\ln \frac{a_{i} \alpha}{S_{s}}-\left(\mu+\sigma^{2}\right) \delta}{\sigma \sqrt{\delta}} ;-\frac{\sqrt{2 \delta-1}}{\sqrt{2 \delta}}\right)\right] .
\end{aligned}
$$

where $p_{1}$ is given in Proposition 1 and $\delta=t-s$. 
Proof. See Appendix A.3

The above results are of paramount importance when investigating the impairment characteristics and thus can be incorporated on a periodic assessment the impact of an impairment of a book of comonotonic equities on the profit sharing mechanism. For instance, this can be integrated on scenario based approach. However, from a risk management point of view it is important to measure the risk of any deviation in the profit or loss resulting from any impairment of such a security. Therefore, in the following result we are looking for the joint market value of an individual stock is the price at which this asset is quoted on an exchange.

Theorem 1 Let $t, x, y \geq 0$. Assume, without loss of generality, that the acquisition costs $a_{i}, i=1 \ldots n$ of $n$ shares are ordered, i.e. $a_{1} \leq a_{2} \cdots \leq a_{n}$. In the case of $n$ shares with different acquisition values $a_{i}$ and different acquisition dates $t_{i}<t, i=1 \ldots n$, the joint c.d.f. $\mathbb{P}_{t}\left(\Gamma_{t+1} \leq y, \Sigma_{t+1} \leq x\right)$ can be written as follows:

$$
\begin{aligned}
& \sqrt{2} \phi\left(\frac{1}{\sigma} \ln \left(\frac{x}{n S_{t}}\right)-\frac{\mu}{\sigma}\right)-\Phi\left(\omega_{n}, \frac{1}{\sigma} \ln \left(\frac{x}{n S_{t}}\right)-\frac{\mu}{\sigma} ; \frac{1}{\sqrt{2}}\right) \\
& +\mathbb{1}_{\frac{x}{n} \leq a_{n} \alpha}\left(\frac{a_{n} \alpha}{S_{t}}\right)^{\frac{2 \mu}{\sigma^{2}}} e^{-\frac{\mu^{2}}{\sigma^{2}}} \Phi\left(\omega_{n}+\frac{2 \mu}{\sigma}, \frac{1}{\sigma} \ln \left(\frac{x S_{t}}{n\left(a_{n} \alpha\right)^{2}}\right)-\frac{\mu}{\sigma} ;-\frac{1}{\sqrt{2}}\right) \\
& +\mathbb{1}_{\frac{x}{n}>a_{n} \alpha}\left\{\Phi\left(\omega_{n}, \frac{1}{\sigma} \ln \left(\frac{x}{n S_{t}}\right)-\frac{\mu}{\sigma} ; \frac{1}{\sqrt{2}}\right)-\Phi\left(\omega_{n}, \frac{1}{\sigma} \ln \left(\frac{a_{n} \alpha}{S_{t}}\right)-\frac{\mu}{\sigma} ; \frac{1}{\sqrt{2}}\right)\right. \\
& \left.+\left(\frac{a_{n} \alpha}{S_{t}}\right)^{\frac{2 \mu}{\sigma^{2}}} e^{-\frac{\mu^{2}}{\sigma^{2}} \Phi}\left(\omega_{n}+\frac{2 \mu}{\sigma},-\frac{1}{\sigma} \ln \left(\frac{a_{n} \alpha}{S_{t}}\right)-\frac{\mu}{\sigma} ;-\frac{1}{\sqrt{2}}\right)\right\} \\
& +\sum_{j=0}^{n-1} \mathbb{1}_{\frac{1}{n-j}\left(\sum_{i=1+j}^{n} a_{i}-y\right) \leq \frac{x}{n} \wedge a_{j+1} \alpha} \times\left\{\Phi\left(\omega_{j+1}, \frac{1}{\sigma} \ln \left(\frac{\frac{x}{n} \wedge a_{j+1} \alpha}{S_{t}}\right)-\frac{\mu}{\sigma} ; \frac{1}{\sqrt{2}}\right)\right. \\
& -\left(\frac{a_{j+1} \alpha}{S_{t}}\right)^{\frac{2 \mu}{\sigma^{2}}} e^{-\frac{\mu^{2}}{\sigma^{2}}} \Phi\left(\omega_{j+1}+\frac{2 \mu}{\sigma}, \frac{1}{\sigma} \ln \left(\frac{S_{t}\left(\frac{x}{n} \wedge a_{j+1} \alpha\right)}{\left(a_{j+1} \alpha\right)^{2}}\right)-\frac{\mu}{\sigma} ;-\frac{1}{\sqrt{2}}\right) \\
& -\Phi\left(\omega_{j+1}, \frac{1}{\sigma} \ln \left(\frac{\sum_{i=j+1}^{n} a_{i}-y}{(n-j) S_{t}}\right)-\frac{\mu}{\sigma} ; \frac{1}{\sqrt{2}}\right) \\
& \left.+\left(\frac{a_{j+1} \alpha}{S_{t}}\right)^{\frac{2 \mu}{\sigma^{2}}} e^{-\frac{\mu^{2}}{\sigma^{2}}} \Phi\left(\omega_{j+1}+\frac{2 \mu}{\sigma}, \frac{1}{\sigma} \ln \left(\frac{S_{t}\left(\sum_{i=1+j}^{n} a_{i}-y\right)}{(n-j)\left(a_{j+1} \alpha\right)^{2}}\right)-\frac{\mu}{\sigma} ;-\frac{1}{\sqrt{2}}\right)\right\}
\end{aligned}
$$

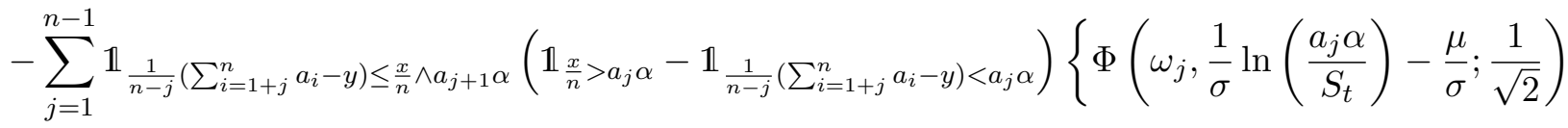

$$
\begin{aligned}
& \left.-\left(\frac{a_{j} \alpha}{S_{t}}\right)^{\frac{2 \mu}{\sigma^{2}}} e^{-\frac{\mu^{2}}{\sigma^{2}}} \Phi\left(\omega_{j}+\frac{2 \mu}{\sigma}, \frac{1}{\sigma} \ln \left(\frac{S_{t}}{a_{j} \alpha}\right)-\frac{\mu}{\sigma} ;-\frac{1}{\sqrt{2}}\right)\right\}
\end{aligned}
$$

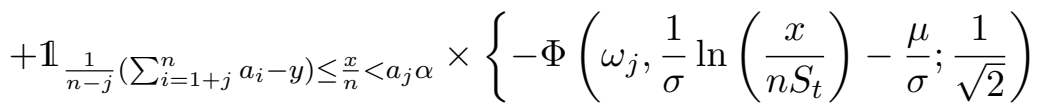

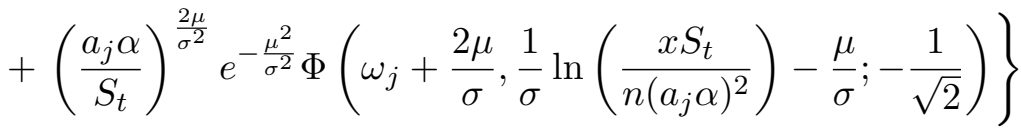




$$
\begin{aligned}
&+\mathbb{1}_{\frac{1}{n-j}}\left(\sum_{i=1+j}^{n} a_{i}-y\right) \leq \frac{x}{n} \wedge a_{j} \alpha\left\{\Phi\left(\omega_{j}, \frac{1}{\sigma} \ln \left(\frac{\sum_{i=1+j}^{n} a_{i}-y}{(n-j) S_{t}}\right)-\frac{\mu}{\sigma} ; \frac{1}{\sqrt{2}}\right)\right. \\
&\left.+\left(\frac{a_{j} \alpha}{S_{t}}\right)^{\frac{2 \mu}{\sigma^{2}}} e^{-\frac{\mu^{2}}{\sigma^{2}}} \Phi\left(\omega_{j}+\frac{2 \mu}{\sigma}, \frac{1}{\sigma} \ln \left(\frac{\left(\sum_{i=1+j}^{n} a_{i}-y\right) S_{t}}{(n-j)\left(a_{j} \alpha\right)^{2}}\right)-\frac{\mu}{\sigma} ;-\frac{1}{\sqrt{2}}\right)\right\},
\end{aligned}
$$

where $\omega_{j}=\frac{\sqrt{2}}{\sigma} \ln \left(a_{j} \alpha / S_{t}\right)+\mu / \sigma \sqrt{2}$.

Proof. See Appendix A.4

From an operational perspective, this first result enables to deal with several lines of a same title which were acquired at different times. Moreover it is already an improvement of the main used approach in cash-flows projection models which consists to aggregate a book of stocks (averaging both market values and acquisition costs) and consider it as if it was a single equity security. Of course this main used approach leads to misrepresent impairment losses dynamic which is far to be linear.

4.2 Shares with linear dependency. In this section, we intend to expand our first result, assuming that the stocks are correlated and they have the following dynamics :

$$
d S_{t}^{i}=S_{t}^{i}\left(r d t+\sigma_{i} d W_{t}^{i}\right)
$$

where $r, \sigma_{i}>0$ and $W^{i}, i=1, \ldots, n$ are $n$ correlated Brownian motions $(\mathrm{BM}), \operatorname{cov}\left(W_{t}^{i}, W_{t}^{j}\right)=\rho_{i j} t$ with $\left.\rho_{i j} \in\right]-1,1\left[\right.$. In this section, $\mathcal{F}_{t}$ is the filtration generated by the $n$ BMs.

Since the expected impaired amount does not depend on the correlation coefficients, we easily find the following result as in the previous section.

Proposition 4 For $n$ correlated shares with different acquisition dates $t_{i}$ and acquisition costs $a_{i}$, the next period expected impaired amount is given as follows for $t_{i} \leq s<t-\frac{1}{2}, i=1 \ldots n$ and $t>\frac{1}{2}$ :

$$
\begin{aligned}
\mathbb{E}_{s}\left(\Gamma_{t}\right) & =\sum_{i=1}^{n} a_{i}\left(1-p_{1}\left(a_{i}, S_{s}^{i}\right)\right) \\
& +\sum_{i=1}^{n} e^{r \delta}\left[-S_{s}^{i} \Phi\left(\frac{\ln \frac{a_{i} \alpha}{S_{s}^{i}}-\left(\mu_{i}+\sigma_{i}^{2}\right)\left(\delta-\frac{1}{2}\right)}{\sigma_{i} \sqrt{\delta-\frac{1}{2}}}, \frac{\ln \frac{a_{i} \alpha}{S_{s}^{i}}-\left(\mu_{i}+\sigma_{i}^{2}\right) \delta}{\sigma_{i} \sqrt{\delta}} ; \frac{\sqrt{2 \delta-1}}{\sqrt{2 \delta}}\right)\right. \\
& \left.+\left(a_{i} \alpha\right) \frac{2\left(\mu_{i}+\sigma_{i}^{2}\right)}{\sigma_{i}^{2}} S_{s}^{-\frac{2 r}{\sigma_{i}^{2}}} \Phi\left(\frac{\ln \frac{a_{i} \alpha}{S_{s}^{i}}+\left(\mu_{i}+\sigma_{i}^{2}\right)\left(\delta-\frac{1}{2}\right)}{\sigma_{i} \sqrt{\delta-\frac{1}{2}}}, \frac{-\ln \frac{a_{i} \alpha}{S_{s}^{i}}-\left(\mu_{i}+\sigma_{i}^{2}\right) \delta}{\sigma_{i} \sqrt{\delta}} ;-\frac{\sqrt{2 \delta-1}}{\sqrt{2 \delta}}\right)\right] .
\end{aligned}
$$

where $p_{1}$ is given in Proposition 1 and $\delta=t-s$.

Henceforth we consider the case of two correlated stocks (i.e. $n=2$ ) where the correlation coefficient between the two Brownian motions is $\rho \in]-1,1[$. We introduce the following key functions needed throughout the sequel. 
Definition 1 Let $\beta \in[0, \pi], \sigma_{1}, \sigma_{2}>0, \mu_{1}, \mu_{2} \in \mathbb{R}$ some constants and $q: \mathbb{R}_{+} \times[0, \beta] \times \mathbb{R}_{+}^{3} \rightarrow[0,1]$, $p: \mathbb{R}_{+}^{2} \times[0, \beta]^{2} \times \mathbb{R}_{+}^{3} \rightarrow[0,1]$ two functions defined by

$$
\begin{aligned}
& q\left(r_{0}, \theta_{0}, t ; M_{1}, M_{2}\right)=e^{M_{1} \alpha_{1}+M_{2} \alpha_{2}+b t} \frac{2}{\beta t} \times \\
& \sum_{n=1}^{\infty} e^{-\frac{r_{0}^{2}}{2 t}} \sin \left(\frac{n \pi \theta_{0}}{\beta}\right) \int_{0}^{\beta} \sin \left(\frac{n \pi \tilde{\theta}}{\beta}\right) \int_{0}^{\infty} \tilde{r} e^{-\frac{\tilde{r}^{2}}{2 t}} e^{\frac{\mu_{2}}{\sigma_{2}} \tilde{r} \sin \theta+\alpha_{1} \sigma_{1} \sqrt{1-\rho^{2}} \tilde{r} \cos \theta} I_{\frac{n \pi}{\beta}}\left(\frac{\tilde{r} r_{0}}{t}\right) d \tilde{r} d \tilde{\theta} \\
& p\left(\tilde{r}, r_{0}, \tilde{\theta}, \theta_{0}, t ; M_{1}, M_{2}\right)=e^{M_{1} \alpha_{1}+M_{2} \alpha_{2}+b t+\frac{\mu_{2}}{\sigma_{2}} \tilde{r} \sin \tilde{\theta}+\alpha_{1} \sigma_{1} \sqrt{1-\rho^{2}} \tilde{r} \cos \tilde{\theta}} \times \\
& \frac{2 \tilde{r}}{\beta t} \sum_{n=1}^{\infty} e^{-\frac{r_{0}^{2}+\tilde{r}^{2}}{2 t}} \sin \left(\frac{n \pi \theta_{0}}{\beta}\right) \sin \left(\frac{n \pi \tilde{\theta}}{\beta}\right) I_{\frac{n \pi}{\beta}}\left(\frac{\tilde{r} r_{0}}{t}\right),
\end{aligned}
$$

where $I_{n}$ is the modified Bessel function,

$$
\alpha_{1}=\frac{-\mu_{1} \sigma_{2}+\rho \mu_{2} \sigma_{1}}{\left(1-\rho^{2}\right) \sigma_{1}^{2} \sigma_{2}}, \alpha_{2}=\frac{-\mu_{2} \sigma_{1}+\rho \mu_{1} \sigma_{2}}{\left(1-\rho^{2}\right) \sigma_{1} \sigma_{2}^{2}}, \text { and } b=\frac{-\mu_{1}^{2} \sigma_{2}^{2}-\mu_{2}^{2} \sigma_{1}^{2}+2 \rho \mu_{1} \mu_{2} \sigma_{1} \sigma_{2}}{2\left(1-\rho^{2}\right) \sigma_{1}^{2} \sigma_{2}^{2}} .
$$

Since in the sequel we consider the case $n=2$, then $\mathcal{F}_{t}$ is the filtration generated by the two Brownian motions $W^{i}, i=1,2$.

If we still intend to express the joint conditional distribution of $(\Gamma, \Sigma)$, the first step leads to express to probability that, at least, one title among the book of assets is impaired.

Proposition 5 Let $t>\frac{1}{2}$. The next period probability of recording an impairment on a given book of 2 correlated shares with acquisition dates $t_{i}$ and acquisition costs $a_{i}, t_{i} \leq s<t-\frac{1}{2}, i=1,2$ is given by

$$
\mathbb{P}_{s}\left(\Gamma_{t}>0\right)=\sum_{i=1}^{2}\left(1-p_{1}\left(a_{i}, S_{s}^{i}\right)\right)-Q\left(S_{s}^{1}, S_{s}^{2}, \delta\right)
$$

where

$$
Q\left(S_{s}^{1}, S_{s}^{2}, \delta\right)=\mathbb{E}\left[\mathbb{1}_{X \leq A_{1}, Y \leq A_{2}} q\left(r_{0}, \theta_{0}, \frac{1}{2} ; \sigma_{1} \sqrt{\delta-\frac{1}{2}}\left(A_{1}-X\right), \sigma_{2} \sqrt{\delta-\frac{1}{2}}\left(A_{2}-Y\right)\right)\right]_{s_{i}=S_{s}^{i}}
$$

$q$ is given in Definition 1, $p_{1}$ is given Proposition 1. Here, $(X, Y)^{t}$ is a Gaussian vector $\mathcal{N}\left(0_{\mathbb{R}^{2}},\left(\begin{array}{cc}1 & \rho \\ \rho & 1\end{array}\right)\right)$ and

$$
\begin{aligned}
r_{0} & =\left(\frac{\delta-\frac{1}{2}}{1-\rho^{2}}\left(\frac{\left(A_{1}-X\right)^{2}}{2}-2 \rho\left(A_{1}-X\right)\left(A_{2}-Y\right)+\frac{\left(A_{2}-X\right)^{2}}{2}\right)\right)^{\frac{1}{2}} \\
\tan \beta & =-\frac{\sqrt{1-\rho^{2}}}{\rho}, \quad \beta \in[0, \pi], \quad \tan \theta_{0}=\sqrt{1-\rho^{2}}\left(\frac{A_{1}-X}{A_{2}-Y}-\rho\right)^{-1}, \quad \theta_{0} \in[0, \beta] \\
A_{i} & =\frac{\ln \frac{a_{i} \alpha}{s_{i}}-\mu_{i}\left(\delta-\frac{1}{2}\right)}{\sigma_{i} \sqrt{\delta-\frac{1}{2}}}, \quad \delta=t-s .
\end{aligned}
$$


Proof. See Appendix B.1

The $Q$ function previously introduced is going to be used to express the conditional distribution of $(\Gamma, \Sigma)$ in 2.

Theorem 2 In the case of two correlated shares $S^{1}$ and $S^{2}$, the joint c.d.f. $\mathbb{P}_{t}\left(\Gamma_{t+1} \leq y, \Sigma_{t+1} \leq x\right)$ can be written as follows for all $x, y \geq 0$ :

$$
\begin{aligned}
& \sum_{i, j \in\{1,2\}, i \neq j} \mathbb{E}\left[J_{i j}^{1}(X, Z) \phi_{i j}\left(s_{1}, s_{2}, X, Y, Z\right)\right]_{s_{i, j}=S_{t}^{i, j}}-\left(\frac{\alpha a_{i}}{S_{t}^{i}}\right)^{\frac{2 \mu_{i}}{\sigma_{i}^{2}}} e^{-\frac{\mu_{i}^{2}}{\sigma_{i}^{2}}} \mathbb{E}\left[J_{i j}^{2}(X, Z) \tilde{\phi}_{i j}\left(s_{1}, s_{2}, X, Y, Z\right)\right]_{s_{i, j}=S_{t}^{i, j}} \\
& +\mathbb{E}\left[\mathbb{1}_{X \leq \frac{\ln \frac{x}{s_{1}}-\mu_{1}}{\sigma_{1}}} \phi\left(\frac{1}{\sigma_{2} \sqrt{1-\rho^{2}}} \ln \frac{x-s_{1} e^{\mu_{1}+\sigma_{1} X}}{s_{2} e^{\mu_{2}+\sigma_{2} \rho X}}\right)\right]_{s_{1,2}=S_{t}^{1,2}} J_{3}+Q\left(S_{t}^{1}, S_{t}^{2}, 1\right) J_{4} \\
& +\int_{0}^{\infty} \int_{0}^{\beta} \mathbb{E}_{t}\left[\mathbb{1}_{X \leq A_{1}, Y \leq A_{2}} p\left(\tilde{r}, r_{0}, \tilde{\theta}, \theta_{0}, \sigma_{1} \sqrt{0.5}\left(A_{1}-X\right), \sigma_{2} \sqrt{0.5}\left(A_{2}-Y\right)\right)\right]_{s_{1,2}=S_{t}^{1,2}} J(\tilde{r}, \tilde{\theta}) d \tilde{r} d \tilde{\theta}
\end{aligned}
$$

where $Q, r_{0}, \theta_{0}, A_{i}($ for $\delta=1)$ and $\beta$ are given in Proposition $5,(X, Y, Z)^{t}$ is a Gaussian vector $\mathcal{N}\left(0_{\mathbb{R}^{3}},\left(\begin{array}{lll}1 & \rho & 0 \\ \rho & 1 & 0 \\ 0 & 0 & 1\end{array}\right)\right)$ and

$$
\begin{aligned}
& J_{i j}^{1}(X, Z)=\mathbb{1}_{X \leq A_{i}}\left[\begin{array}{c}
I_{1}\left(a_{i}, a_{j}\right) \mathbb{1} \\
Z \leq \frac{\ln \frac{x \wedge\left(\alpha a_{i}\right)}{s_{i}}-\mu_{i}}{\sigma_{i} \sqrt{0.5}}-X
\end{array}+I_{2}\left(a_{i}, a_{j}\right) \mathbb{1}_{\frac{\ln \frac{a_{i}-y}{s_{i}}-\mu_{i}}{\sigma_{i} \sqrt{0.5}}-X \leq Z \leq \frac{\ln \frac{x \wedge\left(\alpha a_{i}\right)}{s_{i}}-\mu_{i}}{\sigma_{i} \sqrt{0.5}}-X}\right], \\
& J_{i j}^{2}(X, Z)=\mathbb{1}_{X \leq A_{i}}\left[I_{1}\left(a_{i}, a_{j}\right) \mathbb{1}_{\frac{\ln \frac{a_{i}-y}{s_{i}}-\mu_{i}}{\sigma_{i} \sqrt{0.5}}-X \leq Z \leq \frac{\ln \frac{x \wedge\left(\alpha a_{i}\right)}{s_{i}}-\mu_{i}}{\sigma_{i} \sqrt{0.5}}-X}+I_{2}\left(a_{i}, a_{j}\right) \mathbb{1}_{\ln \frac{s_{i}\left(a_{i}-y\right)}{\left(\alpha a_{i}\right)^{2}}}+X \leq Z \leq \frac{\ln \frac{s_{i}\left(x \wedge \alpha a_{i}\right)}{\left(\alpha a_{i}\right)^{2}}}{\sigma_{i} \sqrt{0.5}}+X\right], \\
& I_{1}\left(a_{i}, a_{j}\right)=-\mathbb{1}_{x \geq \alpha\left(a_{i}+a_{j}\right)} \mathbb{1}_{y<a_{i}}-\mathbb{1}_{y \geq a_{j}} \mathbb{1}_{\alpha a_{i} \leq x<\alpha\left(a_{i}+a_{j}\right)}, \\
& I_{2}\left(a_{i}, a_{j}\right)=\mathbb{1}_{a_{i}(1-\alpha) \leq y<a_{i}} \mathbb{1}_{x \geq\left(\alpha a_{j}\right) \vee\left(a_{i}-y\right)}, \quad i, j \in\{1,2\}, i \neq j, \\
& J_{3}=\mathbb{1}_{x \geq \alpha\left(a_{1}+a_{2}\right)}\left[1+\mathbb{1}_{y \geq a_{1}} \mathbb{1}_{x \geq \alpha a_{2}}+\mathbb{1}_{y \geq a_{2}} \mathbb{1}_{x \geq \alpha a_{1}}\right], \\
& J_{4}=\mathbb{1}_{x \geq \alpha\left(a_{1}+a_{2}\right)}\left[\mathbb{1}_{y<a_{1}}-\mathbb{1}_{a_{2} \leq y<a_{1}+a_{2}}\right] \text {, } \\
& J(\tilde{r}, \tilde{\theta})=-I_{2}\left(a_{1}, a_{2}\right) \mathbb{1}_{(\tilde{r}, \tilde{\theta}) \in D_{1}}-I_{2}\left(a_{2}, a_{1}\right) \mathbb{1}_{(\tilde{r}, \tilde{\theta}) \in D_{2}}+\mathbb{1}_{x \geq\left(a_{1}+a_{2}-y\right) \vee \alpha\left(a_{1}+a_{2}\right)} \mathbb{1}_{y<a_{1}+a_{2}} \mathbb{1}_{(\tilde{r}, \tilde{\theta}) \in D_{3}\left(a_{1}+a_{2}-y\right)} \\
& +\mathbb{1}_{x<\alpha\left(a_{1}+a_{2}\right)} \mathbb{1}_{y \geq a_{1}+a_{2}} \mathbb{1}_{(\tilde{r}, \tilde{\theta}) \in D_{4}(x)}+\mathbb{1}_{0<a_{1}+a_{2}-y \leq x<\alpha\left(a_{1}+a_{2}\right)} \mathbb{1}_{(\tilde{r}, \tilde{\theta}) \in D_{3}(x)-D_{3}\left(a_{1}+a_{2}-y\right)},
\end{aligned}
$$

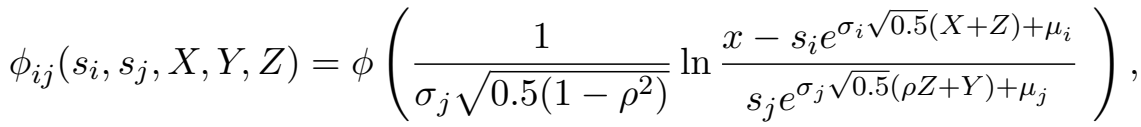

$$
\begin{aligned}
& \tilde{\phi}_{i j}\left(s_{i}, s_{j}, X, Y, Z\right)=\phi\left(\frac{1}{\sigma_{j} \sqrt{0.5\left(1-\rho^{2}\right)}} \ln \frac{x-\frac{\left(\alpha a_{i}\right)^{2}}{s_{i}} e^{\sigma_{i} \sqrt{0.5}(Z-X)}}{\left(\frac{\alpha a_{i}}{s_{i}}\right)^{2 \rho \sigma_{j} / \sigma_{i}} s_{j} e^{\sigma_{j} \sqrt{0.5}(\rho Z+Y-\rho X)+\mu_{j}-2 \rho \mu_{i} \frac{\sigma_{j}}{\sigma_{i}}}}\right), \\
& D_{1}=\left\{(\tilde{r}, \tilde{\theta}): a_{1} \alpha e^{-\sigma_{1} \tilde{r}\left(\rho \sin (\tilde{\theta})+\sqrt{1-\rho^{2}} \cos (\tilde{\theta})\right)}+a_{2} \alpha e^{-\sigma_{2} \tilde{r} \sin (\tilde{\theta})} \leq x, 1 \geq e^{-\sigma_{1} \tilde{r}\left(\rho \sin (\tilde{\theta})+\sqrt{1-\rho^{2}} \cos (\tilde{\theta})\right)} \geq \frac{a_{1}-y}{\alpha a_{1}}\right\},
\end{aligned}
$$




$$
\begin{aligned}
& D_{2}=\left\{(\tilde{r}, \tilde{\theta}): a_{1} \alpha e^{-\sigma_{1} \tilde{r}\left(\rho \sin (\tilde{\theta})+\sqrt{1-\rho^{2}} \cos (\tilde{\theta})\right)}+a_{2} \alpha e^{-\sigma_{2} \tilde{r} \sin (\tilde{\theta})} \leq x, 1 \geq e^{-\sigma_{2} \tilde{r} \sin (\tilde{\theta})} \geq \frac{a_{2}-y}{\alpha a_{2}}\right\}, \\
& D_{3}(m)=\left\{(\tilde{r}, \tilde{\theta}): a_{1} \alpha e^{-\sigma_{1} \tilde{r}\left(\rho \sin (\tilde{\theta})+\sqrt{1-\rho^{2}} \cos (\tilde{\theta})\right)}+a_{2} \alpha e^{-\sigma_{2} \tilde{r} \sin (\tilde{\theta})} \leq m\right\}, \quad \forall m \geq 0 .
\end{aligned}
$$

Proof. See Appendix B.2.

Notice that our method can be generalized to $n>2$ if the joint density $(X, \tilde{X})$ is known, with $X=\left(X^{1}, \ldots, X^{n}\right)$ is a $n$-dimensional drifted Brownian motion and $\tilde{X}=\left(\tilde{X}^{1}, \ldots, \tilde{X}^{n}\right)$ its element-wise supremum over a given period. To our knowledge, this density only exists for some particular correlation matrices, see Escobar and Hernandez (2014). The determination of such correlation structures is based on the method of images, see Keller (1953). In our case, we note $R$ the correlation matrix of the vector $X$ and introduce the matrices $A_{n}, B_{n}$ and $C_{n}$ that will needed to characterize the structures that can lead to the derivation of the density depending on the dimension $n$. The elements of these three matrices are given as follows:

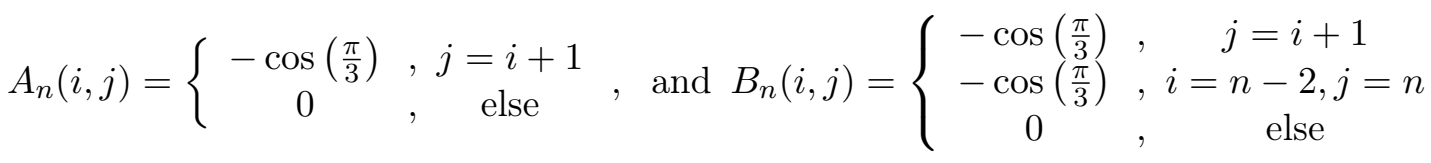

$$
\begin{aligned}
& C_{n}(i, j)=\left\{\begin{array}{cc}
-\cos \left(\frac{\pi}{3}\right) & , \quad j=i+1 \\
-\cos \left(\frac{\pi}{4}\right) & , i=n-1, j=n \\
0 & , \quad \text { else. }
\end{array}\right.
\end{aligned}
$$

Theorem 3 The type of correlation matrices $R$ where the method of images can be used to find the joint distribution of $(X, \tilde{X})$ is provided next :

1. If $n=3$, then there are only three cases : $A_{3}, C_{3}$ and $G_{3}$ where

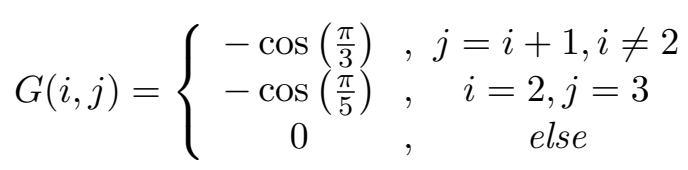

2. For any $n>3$ there are at least 3 cases of correlation matrices: $A_{n}, B_{n}, C_{n}$.

(a) If $n=4$ then there are a total of 5 cases : $A_{4}, B_{4}, C_{4}$ and $F_{4}, G_{4}$ where

$$
F_{4}(i, j)=\left\{\begin{array}{cc}
-\cos \left(\frac{\pi}{3}\right), & j=i+1, i \neq 2 \\
-\cos \left(\frac{\pi}{4}\right), & i=2, j=3 \\
0 \quad, & \text { else }
\end{array} \quad \text { and } G_{4}(i, j)=\left\{\begin{array}{cc}
-\cos \left(\frac{\pi}{3}\right), j=i+1, i \neq 3 \\
-\cos \left(\frac{\pi}{5}\right), & i=3, j=4 \\
0 \quad, & \text { else }
\end{array}\right.\right.
$$

(b) If $n=5$ then there are only three cases: $A_{5}, B_{5}, C_{5}$.

(c) If $n=6,7$ or 8 , then there are 4 cases: $A_{n}, B_{n}, C_{n}$ and $E_{n}$ where

$$
E_{n}(i, j)=\left\{\begin{array}{ccc}
-\cos \left(\frac{\pi}{3}\right) & , j=i+1, i \neq 4 \\
0 & , \quad i=4, j=5 \\
-\cos \left(\frac{\pi}{3}\right), & i=3, j=5 \\
0 & , & \text { else. }
\end{array}\right.
$$


Let's note that in the case $n=2$ we use the joint density of $(X, \tilde{X})$ to find $\mathbb{P}_{t}\left(\Gamma_{t+1} \leq y, \Sigma_{t+1} \leq x\right)$, but the c.d.f of this vector is enough for calculations.

Remark 1 If we add a dependent share to a set of comonotonic stocks, we can easily derive analytical results as above. In fact, the triggering events of the comonotonic shares reduce to exploring a single random process driving these shares. Thus, the calculation in presence of a dependent share follows similar steps as detailed above while taking into account the correlation between the two Brownian motions.

\section{Operational implementation}

In this section, we illustrate the computation of the provisions and compare the closed form formulas to the simulated approximations. For the sake of readiness we consider the case where the insurer holds two assets, i.e. $n=2$, for which we run Monte-Carlo experiments to quantify the quantities introduced in the previous sections. To this end, we adopt a naïve approach to implement the simulation scheme. Let $S_{t_{k}}^{M, i}$ be the realizations of the dynamics of the process $\left(S_{t}\right)_{t \geq 0}$ over a discrete-time grid $t_{k}, k=0,1, \cdots, M$, of length $\Delta t=t_{k}-t_{k-1}=1 / M$ with

$$
S_{t_{k}}^{M, i}=S_{t_{k-1}}^{M, i} \exp \left(\mu^{i} \Delta t+\sigma^{i} \sqrt{\Delta t} X_{k}\right)
$$

where $X_{k}$ are independent realizations of a standard normal r.v. $X$. Accordingly, a realization of the maximum over $[t+1 / 2, t]$ can be approximated by

$$
\widetilde{S}_{t}^{M, i}=\max \left\{S_{t_{k}}^{M, i}, t_{k} \in[t+1 / 2, t+1], k=0,1, \cdots\right\} .
$$

Using this path approximation, we run standard Monte-Carlo approach to simulate the impairment $\operatorname{PDD}_{t}^{i}$ in Equation (2.2) for a given observation time $t$. More formally, let $n=2$ then for $i=1$, 2 , we have the following approximation for the future impairment:

$$
\begin{aligned}
\Sigma_{t_{N}}^{M} & =S_{t_{N}}^{M, 1}+S_{t_{N}}^{M, 2} \\
\Gamma_{t_{N}}^{M} & =\left(S_{t_{1}}^{M, 1}-S_{t_{N}}^{M, 1}\right)^{+} \mathbb{1}_{\widetilde{S}_{t_{N}}^{M, 1} \leq \alpha S_{t_{1}}^{M, 1}}+\left(S_{t_{2}}^{M, 2}-S_{t_{N}}^{M, 2}\right)^{+} \mathbb{1}_{\widetilde{S}_{t_{N}}^{M, 2} \leq \alpha S_{t_{2}}^{M, 2}} .
\end{aligned}
$$

These are used to approximate the different quantities considered in the above sections and will be compared to the proposed formulas on a number of examples. The simulation experiments were performed on a mac-OS with an Intel Core i7 $2.80 \mathrm{GHz}$ processor.

5.1 Comonotonic shares. We consider two lines of shares with the same underlying security $S^{1}$. The latter was acquired at two different dates $t_{1}$ and $t_{2}$ at cost $a_{1}$ and $a_{2}$. This makes the impairment applicable considering the two shares separately but with the same underlying dynamics. Hereafter, we omit the accumulated impairment losses and thus the carrying amount is equal to the cost (purchase) amount. For illustration, we consider the impairment at terminal period $t=1$ and the quantification from the initial time $s=0$. The security has an initial market value $S_{0}^{1}$ and a given implied volatility $\sigma_{1}$. The prolonged component of the impairment is characterized at a prefixed level $\alpha=80 \%$. Figures 1 and 2 depict the evolution of the next-period impairment probability as well as the expected impaired amount as a function of some key parameters. Here, we used $N=20000$ replications on a discretized time-grid $t_{k}=k \Delta t$ with $M(100$ and 1000) equidistant steps. 

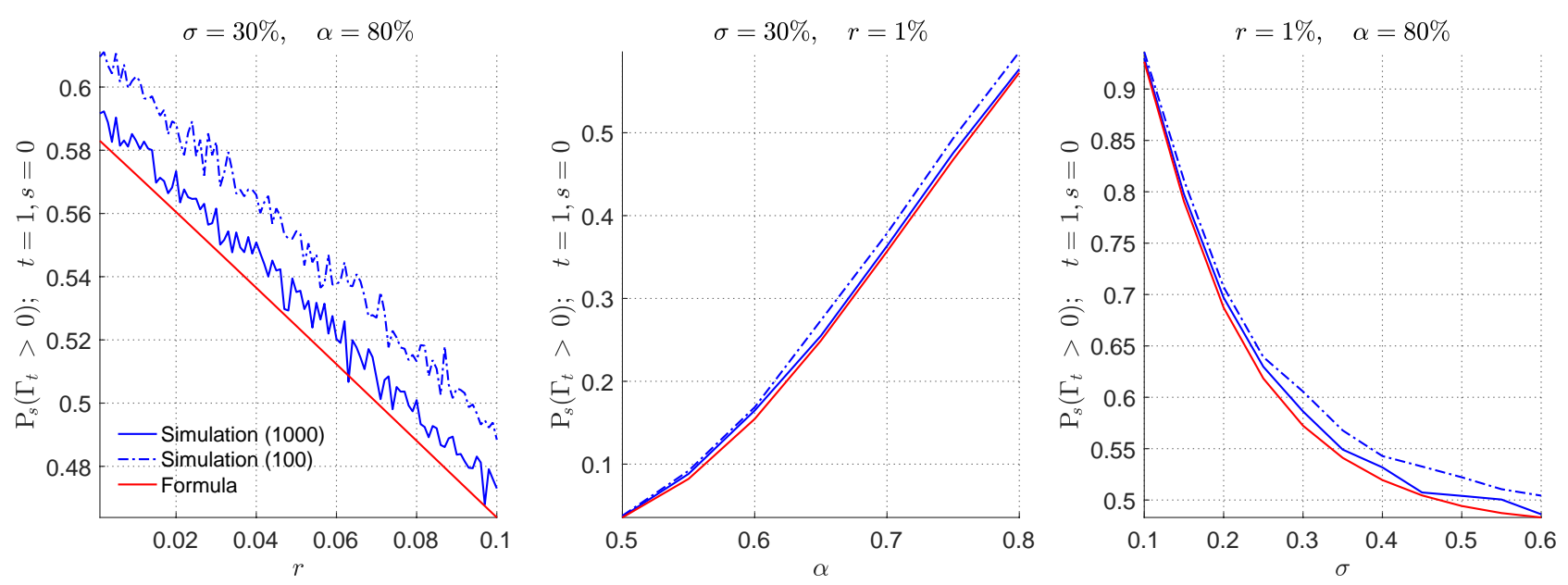

Figure 1: Distribution function. The probability $\mathbb{P}_{s}\left(\Gamma_{t}>0\right)$ in Proposition 1, for $t=1$ ( $\left.s=0\right)$ as a function of the risk-free interest rate $r$ (left), the level $\alpha$ (middle) and the volatility $\sigma$ (right).

Figure 2 illustrates the expected impairment in Proposition 3. We observe that when the risk-free return $r$ is high the impairment loss will be approaching zero almost surely, see Figure 1 for the corresponding probabilities. This is of course related to the initial buffer of capital gains, which is assumed to be zero for the base case. If the initial impairment is not omitted, one should expect a less of an impact. In the other hand, a volatility increase leads to the rise of the impaired value and thus can reach $45 \%$ of the initial cost. Regarding the simulated approximations (in blue), we remark that those are fairly reproducing the exact results with respect to the exact computations. This comparison demonstrates that the Monte-Carlo estimation is a biased estimator (the exact value is inside the 0.95 confidence interval of the estimates for any $M)$. However, we remark that the estimate based on a discretely monitored prolonged constraint (barrier-type condition), see the approximation in Equation (5.1) converges to the continuous case (red line) as $M$ increases. However, the convergence is very slow and the bias is larger than $5 \%$ of the true values (both for the c.d.f. and the expected value) even for 1000 time steps. Therefore, the use of the analytical formulas are very efficient as the number of required amount of sampling dates and simulations to achieve a descent approximation is high, see Figure 2 and Figure 1. In fact, this is due to the error coming from the discretization of the dynamics in Equation (4.3) and especially the approximation of the maximum in Equation (5.1). More precisely, there is a loss of information about all parts of the continuous-time path between sampling dates, which introduces a substantial bias of estimation. Moreover, the latter decreases very slowly as $1 / \sqrt{M}$, see Gobet (2000). In order to enhance this bias we can use a discretization scheme based on the exact conditional law of the maximum. The technique is very efficient because only two times steps $\left(t_{1}=1 / 2\right.$ and $\left.t_{2}=1\right)$ are required to simulate the equity path and its supremum is drawn based on the simulation of a one-dimensional Brownian bridge supremum between the sampled dates using its distribution. This technique outperforms the naïve Euler scheme presented above and reduces the bias as well as the convergence rate, see Gobet (2000).

In Table 1 and Table 2 we reported the CPU times of the simulations for $N=1000,5000$ and 10000 and 100 and 1000 numbers of time steps. We display the analytical (true) value for the expected impairment at the end of the first period (lower panel) as well as the probability of positive impairment (left panel). The choice of the number of paths is an important issue here. In fact, the number of steps is of little impact especially when looking at the variance of the estimations. The latter is almost equivalent for 

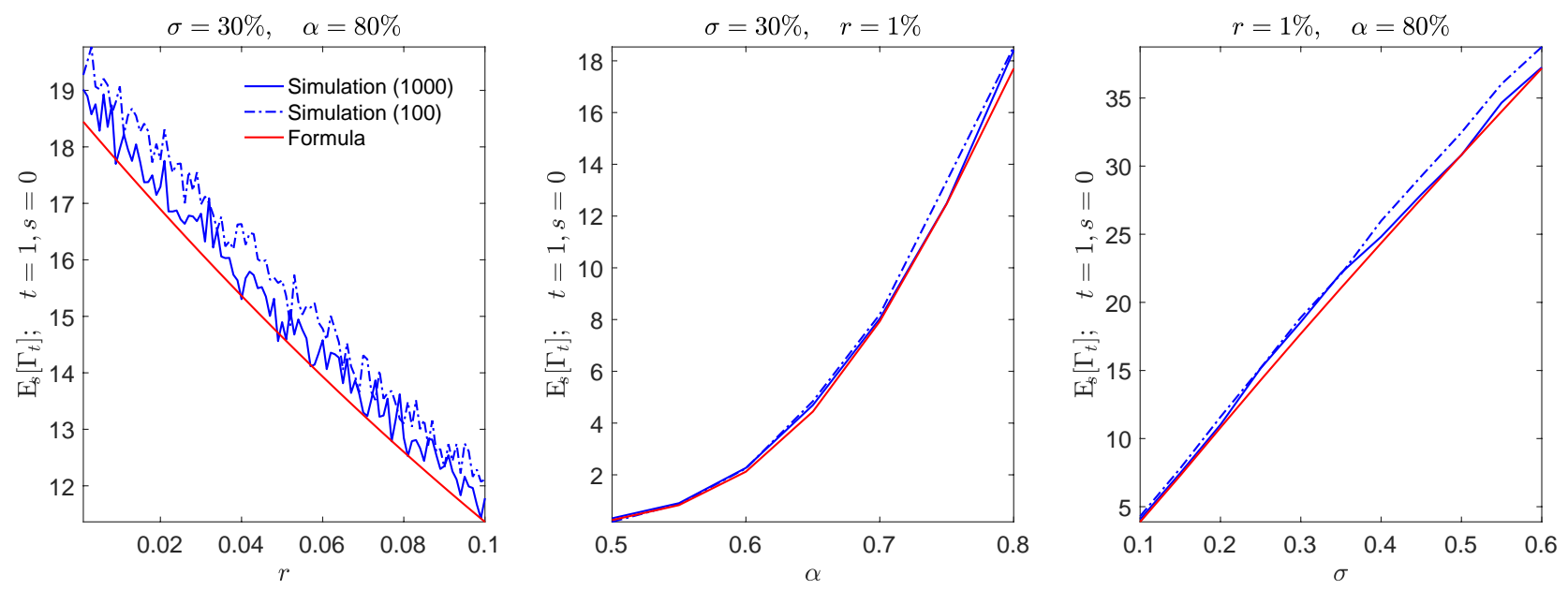

Figure 2: Expected impairment. The conditional first moment $\mathbb{E}_{s}\left(\Gamma_{t}\right)$ in Proposition 3, for $t=1$ $(s=0)$ as a function of the risk-free interest rate $r$ (left), the level $\alpha$ (middle) and the volatility $\sigma$ (right).

different time steps but decreases as the number of simulations increases. However, this bias reduction on the estimated quantities increases the computational time. For instance, the 10000 simulations scheme increases the accuracy up to ten times compared to 1000 scheme but does increase the computational time by the same rate.

In the left panel of Figure 4 in the Appendix C, we plotted the joint distribution of the aggregated equities and the corresponding impairment. We used the analytical formula given in Theorem 1 for $n=2$ and different values of the input parameters. We observe that the probability to recognize an impairment next year together with a fall of the underlying equities does not exhibit a particular pattern: neither globally convex in $x$ and $y$ nor concave. This probability is very low for some levels of $x$ and $y$, which is intuitively predictable. However, for such levels the simulation based estimation, see Figure 5 in the Appendix C, had difficulties to capture these joint probabilities as the variance of the estimation is very high. In fact, even if the number of simulated paths is sufficiently high, the scenarios triggering the events (the impairment together with the equities drop-down) are rare and thus make the estimation unstable and volatile.

5.2 Correlated securities. The case of correlated assets relies on semi analytical formulas that require further computations. Indeed, the results in Subsection 4.2, i.e. Proposition 5 and Theorem 2, involve not only some expectations but also multiple improper integrals. However, the underlying r.v. have a standard normal density and should not be of a particular concern for the implementation. Also, we should note that these formulas can be simplified for some particular forms of the correlation coefficient $\rho$. Indeed, when the latter can take on only the special values $\rho_{k}=-\cos (\pi / k), k=1,2, \cdots$, the two functions $q$ and $p$ in Definition 1 can be determined in a more tractable form. In particular, the infinite sum involved in the definition of $p$ can be written as a finite sum of bivariate normal densities. A similar finite sum formula can be derived for the terms involved in the joint density, see He et al. (1998) for instance and Lemma 2 in Appendix B. Still, the analytical form involves some expectations. Therefore, we focus on the execution time needed to implement each of these quantities as well as the underlying bias.

For the sake of simplicity, we will focus on the above mentioned special case and let the correlation coefficient equal to $\rho_{k}=-\cos (\pi / k)$. For instance, we consider the case where $k=5$ and look at the c.d.f. 
Table 1: Computational Time (Probability). The calculation time and variance of the estimation for the impairment probability $\mathbb{P}_{s}\left(\Gamma_{t}>0\right)$ in Proposition 2 and Proposition 5 with $t=1$ and $s=0$ for two shares $(n=2)$. The input parameters are as follows $\alpha=80 \%, r=1 \%$.

Comonotonic shares (left panel): $S_{0}^{1}=100$ and acquisition costs $S_{t_{1}}^{1}=120, S_{t_{2}}^{1}=150$ and $\sigma_{1}=30 \%$. Correlated shares (right panel): $S_{0}^{1}=100, S_{0}^{2}=120, S_{t_{1}}^{1}=120, S_{t_{2}}^{2}=150$ and $\sigma_{1}=30 \%, \sigma_{2}=40 \%$.

\begin{tabular}{lccc||lccc}
\hline \multicolumn{1}{c}{ Comonotonic } & \multicolumn{1}{c}{$\|$} & \multicolumn{3}{c}{ Correlated $(k=5)$} \\
\hline$N$ & $\mathbf{1 0 0 0}$ & $\mathbf{5 0 0 0}$ & $\mathbf{1 0 0 0 0}$ & & $\mathbf{1 0 0 0}$ & $\mathbf{5 0 0 0}$ & $\mathbf{1 0 0 0 0}$ \\
\hline Analytical & \multicolumn{3}{c}{$\mathbf{0 . 5 7 2 3}$} & & Analytical & & $\mathbf{0 . 5 3 0 4}$ \\
Time (in seconds) & - & - & - & Time (in seconds) & 0.10 & 0.53 & 2.92 \\
Variance (1e-10) & - & - & - & Variance (1e-10) & 0.284 & 0.054 & 0.014 \\
\hline Simulation ( $M=100)$ & & $\mathbf{0 . 5 9 3 8}$ & & Simulation ( $M=100)$ & & $\mathbf{0 . 5 6 3 7}$ \\
Time (in seconds) & 0.00 & 0.01 & 0.07 & Time (in seconds) & 0.02 & 0.06 & 0.17 \\
Variance (1e-3) & 0.239 & 0.046 & 0.013 & Variance (1e-3) & 0.262 & 0.046 & 0.013 \\
\hline Simulation ( $M=1000)$ & & $\mathbf{0 . 5 8 2 7}$ & & Simulation ( $M=1000)$ & & $\mathbf{0 . 5 4 0 2}$ \\
Time (in seconds) & 0.04 & 0.18 & 0.91 & Time (in seconds) & 0.08 & 0.46 & 2.77 \\
Variance (1e-3) & 0.245 & 0.049 & 0.011 & Variance (1e-3) & 0.264 & 0.045 & 0.012 \\
\hline
\end{tabular}

in Proposition 5. In fact, as mentioned earlier the next-period expected impairment does not depend on the correlation between the two shares and the discussion made in the previous section also applies as one can only simulate comonotonic shares but with different initial values to recover the underlying result for the linearly dependent case. In Figure 3 we depict this c.d.f. for different discretization schemes $(M=100$ and $M=1000)$. As we can see similar conclusions can drawn as for the comonotonic case. Regarding the execution time, see the right panel of Table 1, the semi-analytical can be computationally intensive. However, for small number of paths, $N=1000$, the variance of estimate is $10^{7}$ smaller than a naïve Monte-Carlo scheme variance with an equivalent execution time, i.e. 0.28 seconds.

\section{Concluding Remarks}

In this paper, we considered French impairment rules regarding equity securities held by an insurer. Our objective was to go further than common practice which consists in considering an overall portfolio of equities as a single aggregated stock, since the impairment mechanism is complex and has to be considered on an individual basis. Under the Black-Scholes framework (geometric Brownian motion dynamics for the stocks), we derived closed form formulas for the joint distributions of the impaired amounts and the equities market values as well as the next-period impairment provisions. Formally, the results present analytical formulas for the comonotonic shares and semi-analytical forms in the linearly dependent case. The latter can be improved to obtain more tractable formulas depending on the correlation factor. These results enable to improve cash-flows projection models which are of massive use for both prudential and financial reporting purposes. Moreover, our results are the starting ground for future research on the optimal aggregation of equities. In fact, in future work we intend to study the construction of a proxy for representing a book of equities while ensuring the adequate representation of the distributional behaviour of both the aggregated net accounting values and market values. 
Table 2: Computational Time (Expectation). The calculation time and variance of the estimation for the impairment probability $\mathbb{E}_{s}\left[\Gamma_{t}\right]$ in Proposition 3 with $t=1$ and $s=0$ for two shares $(n=2)$. The input parameters are as follows $\alpha=80 \%, r=1 \%, S_{0}^{1}=100$ and acquisition costs $S_{t_{1}}^{1}=120, S_{t_{2}}^{1}=150$ and $\sigma_{1}=30 \%$.

\begin{tabular}{lccc}
\hline$N$ & $\mathbf{1 0 0 0}$ & $\mathbf{5 0 0 0}$ & $\mathbf{1 0 0 0 0}$ \\
\hline Analytical & \multicolumn{3}{c}{$\mathbf{1 2 . 8 9 6 7}$} \\
\hline Simulation $(M=100)$ & \multicolumn{3}{c}{$\mathbf{1 3 . 4 4 3 4}$} \\
\hline Time (in seconds) & 0.01 & 0.03 & 0.12 \\
Variance & 0.689 & 0.151 & 0.036 \\
\hline Simulation $(M=1000)$ & \multicolumn{3}{c}{$\mathbf{1 3 . 1 8 1 2}$} \\
Time (in seconds) & 0.06 & 0.34 & 1.98 \\
Variance & 0.758 & 0.139 & 0.033 \\
\hline
\end{tabular}

\section{References}

Azzaz, J., Loisel, S., and Thérond, P.-E. (2015). Some characteristics of an equity security next-year impairment. Review of Quantitative Finance and Accounting, 45(1):111-135.

Bennemann, C. and Hennig, C. (2010). Impairment estimates of equity portfolios represented by model points. Belgian Actuarial Bulletin, 9(1):43-49.

Escobar, M. and Hernandez, J. (2014). A note on the distribution of multivariate brownian extrema. International Journal of Stochastic Analysis, 2014.

Gobet, E. (2000). Weak approximation of killed diffusion using euler schemes. Stochastic processes and their applications, 87(2):167-197.

Harrison, M. J. (1985). Brownian Motion and Stochastic Flow Systems. Wiley.

He, H., Keirstead, W., and Rebholz, J. (1998). Double lookbacks. Mathematical Finance, 8(3):201-228.

Keller, J. B. (1953). The scope of the image method. Communications on pure and applied mathematics, 6(4):505512.

Thérond, P.-E. (2016). About Market Consistent Valuation in Insurance. In Laurent, J.-P., Norberg, R., and Planchet, F., editors, Modelling in Life Insurance: A Management Perspective, chapter 2, pages 43-60. Springer.

Vedani, J., El Karoui, N., Loisel, S., and Prigent, J. L. (2017). Market inconsistencies of market-consistent European life insurance economic valuations: pitfalls and practical solutions. European Actuarial Journal, 7(1).

Wüthrich, M. (2016). Market-Consistent Actuarial Valuation. Springer.

\section{Appendices}

\section{A Comonotonic securities}

In order to prove our results, we shall need the joint distribution of a Brownian motion with drift and its supremum. This can be easily derived using Harrison (1985). 

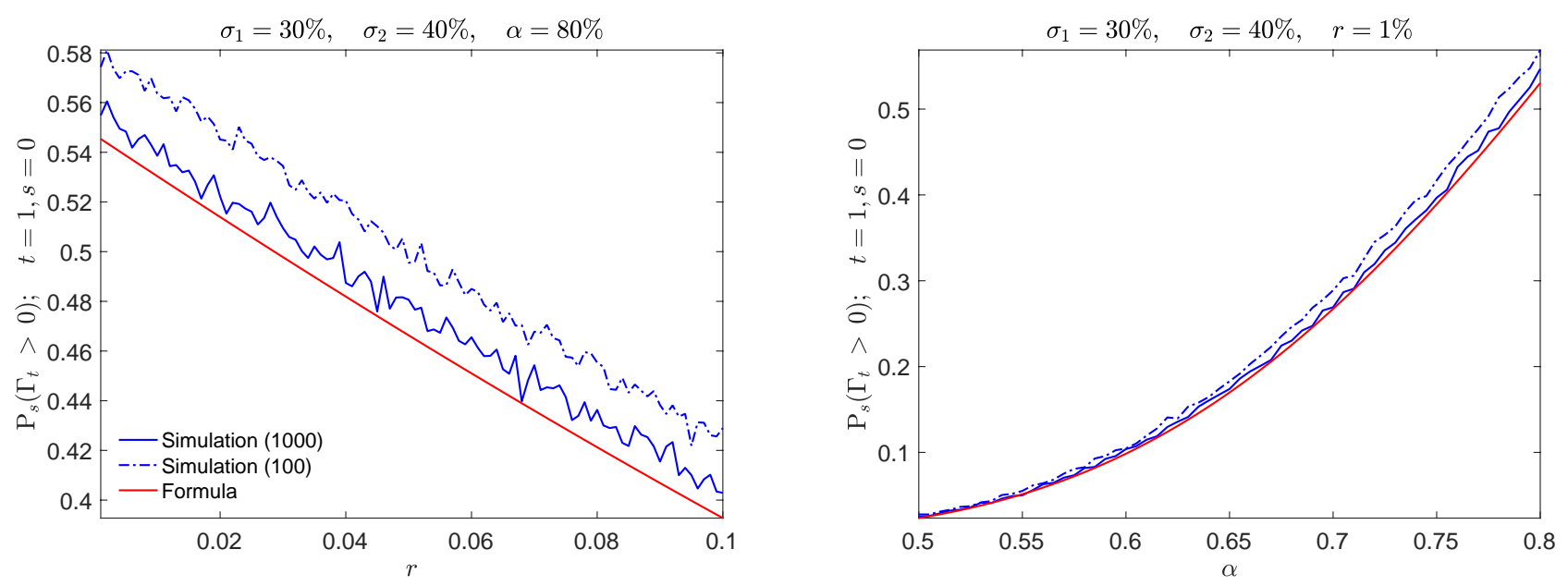

Figure 3: Distribution function. The probability $\mathbb{P}\left(\Gamma_{t}>0\right)$ in Proposition 5 , for $t=1$ and $s=0$, as a function of the interest rate $r$ (left) and impairment level $\alpha$ (right) for equities $S_{0}^{1}=100, S_{0}^{2}=120$ and $S_{t_{1}}^{1}=120$ and $S_{t_{2}}^{2}=150$.

Proposition 6 Let $X_{t}=\mu t+\sigma B_{t}$, and $M_{t}=\sup _{u \in[0, t]} X_{u}$ where $B$ is a standard Brownian motion. Then, the probability $\mathbb{P}\left(X_{t} \leq y, M_{t} \geq x\right)$ is given by:

$$
\begin{cases}\phi\left(\frac{y-\mu t}{\sigma \sqrt{t}}\right) & \text { if } 0 \geq x, \\ e^{2 \mu x / \sigma^{2} \phi\left(\frac{y-2 x-\mu t}{\sigma \sqrt{t}}\right)} & \text { if } 0<x, y \leq x, \\ \phi\left(\frac{y-\mu t}{\sigma \sqrt{t}}\right)-\phi\left(\frac{x-\mu t}{\sigma \sqrt{t}}\right)+e^{2 \mu x / \sigma^{2}} \phi\left(\frac{-x-\mu t}{\sigma \sqrt{t}}\right) & \text { if } 0<x<y .\end{cases}
$$

In the other hand, the c.d.f. $\mathbb{P}\left(M_{t} \leq x\right)$ is given by:

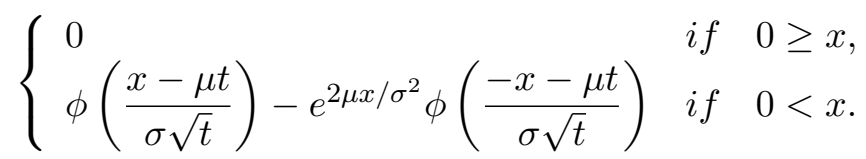

where $\phi$ is the c.d.f of a standard Gaussian r.v.

We easily deduce

$$
\mathbb{P}\left(X_{t} \in d y, M_{t} \leq x\right)=\mathbb{1}_{0<x, y \leq x} \frac{1}{\sigma \sqrt{2 \pi t}}\left(e^{-\frac{(y-\mu t)^{2}}{2 \sigma^{2} t}}-e^{\frac{2 \mu x}{\sigma^{2}}} e^{-\frac{(y-2 x-\mu t)^{2}}{2 \sigma^{2} t}}\right) d y .
$$

Another useful result is the following :

Proposition 7 Let $\alpha, \beta, w, c \in \mathbb{R}$ and $X$ is a standard Gaussian random variable, i.e. $X \sim \mathcal{N}(0,1)$ with distribution function $\phi$. Then

$$
\mathbb{E}\left[\mathbb{1}_{X \leq w} e^{-c X} \phi(a X+b)\right]=e^{c^{2} / 2} \Phi\left(w+c, \frac{b-a c}{\sqrt{1+a^{2}}} ; \frac{-a}{\sqrt{1+a^{2}}}\right)
$$

where $\Phi(U, V ; \theta)$ is the joint c.d.f. of two correlated Gaussian r.v.s $U$ and $V$ with a given correlation coefficient $\theta$. 
Proof. If one considers two correlated random variables $X_{1}$ and $X_{2}$ with correlation coefficient $\rho$ such that $X_{i} \sim \mathcal{N}\left(\mu_{i}, \sigma_{i}\right), i=1,2$ then the distribution of $X_{2}$ conditionally on $X_{1}$ can be written as follows:

$$
X_{2} \mid X_{1} \sim \mathcal{N}\left(\mu_{2}+\rho \frac{\sigma_{2}}{\sigma_{1}}\left(X_{1}-\mu_{1}\right),\left(1-\rho^{2}\right) \sigma_{2}^{2}\right) .
$$

Thus, we can write the joint distribution of $X_{1}$ and $X_{2}$ in the following form

$$
\mathbb{P}\left(X_{1} \leq x_{1}, X_{2} \leq x_{2}\right)=\mathbb{E}\left[\mathbb{1}_{X \leq \frac{x_{1}-\mu_{1}}{\sigma_{1}}} \phi\left(\frac{y-\mu_{2}-\rho \frac{\sigma_{2}}{\sigma_{1}}\left(X \sigma_{1}+\mu_{1}-\mu_{1}\right)}{\sigma_{2} \sqrt{1-\rho^{2}}}\right)\right],
$$

which can also be given in terms of the joint distribution $\Phi$ of two standard Gaussian r.v.'s with a given correlation coefficient. Therefore, we can write for any $a, b, c$ and $w$

$$
\begin{aligned}
\mathbb{E}\left[\mathbb{1}_{X \leq w} e^{-c X} \phi(a X+b)\right] & =e^{c^{2} / 2} \mathbb{E}\left[\mathbb{1}_{X \leq w+c} \phi(\alpha X+b-a c)\right] \\
& =e^{c^{2} / 2} \Phi\left(w+c, \frac{b-a c}{\sqrt{1+a^{2}}} ; \frac{-a}{\sqrt{1+a^{2}}}\right)
\end{aligned}
$$

which is the desired result.

A.1 Proof of Proposition 1. The cases $x<0$ and $x \geq a_{i}$ are obvious. Remark that on $\left\{\tilde{S}_{t}^{i} \leq \alpha a_{i}\right\}$, $a_{i}(1-\alpha) \leq a_{i}-S_{t}^{i}$. Hence, for $x<a_{i}(1-\alpha)$,

$$
\mathbb{P}_{s}\left(\mathrm{PDD}_{t}^{i} \leq x\right)=\mathbb{P}_{s}\left(\tilde{S}_{t}^{i}>a_{i} \alpha\right)=1-\mathbb{P}_{s}\left(\mathbb{P}_{t-\frac{1}{2}}\left(\tilde{S}_{t}^{i} \leq a_{i} \alpha\right)\right)
$$

We then use the Markov property of $S^{i}$ and Proposition 6 .

For $a_{i}(1-\alpha) \leq x<a_{i}$, we have

$$
\begin{aligned}
\mathbb{P}_{s}\left(\mathrm{PDD}_{t}^{i} \leq x\right) & =\mathbb{E}_{s}\left(\left(a_{i}-S_{t}^{i}\right)^{+} \mathbb{1}_{\tilde{S}_{t}^{i} \leq \alpha a_{i}} \leq x\right) \\
& =\mathbb{E}_{s}\left[\mathbb{E}_{t-\frac{1}{2}}\left(\left(a_{i}-S_{t}^{i}\right)^{+} \mathbb{1}_{\tilde{S}_{t}^{i} \leq \alpha a_{i}} \leq x\right)\right]
\end{aligned}
$$

Using the Markov property of $S^{i}$, we get

$$
\mathbb{E}_{s}\left[\mathbb{1}_{S_{t-\frac{1}{2}}^{i} \leq \alpha a_{i}} \mathbb{E}_{t-\frac{1}{2}}\left(\left(a_{i}-S_{t-\frac{1}{2}}^{i} e^{\frac{\mu_{i}}{2}+\sigma_{i} B_{\frac{1}{2}}}\right) \mathbb{1}_{\sup _{u \in\left[0, \frac{1}{2}\right]}\left(\mu_{i} u+\sigma_{i} B_{u}\right) \leq \ln \left(a_{i} \alpha / S_{t-1 / 2}^{i}\right)} \leq x\right)\right],
$$

where $B$ is a standard Brownian motion independent of $W$. We firstly use Proposition 6 to compute the conditional expectation and secondly we remark that $S_{t-\frac{1}{2}}^{i}=S_{s}^{i} e^{X_{\delta-\frac{1}{2}}}$ where $X_{\delta-\frac{1}{2}}=\mu_{i}\left(\delta-\frac{1}{2}\right)+$ $\sigma_{i} \sqrt{\delta-\frac{1}{2}} X$ where $X$ is a standard Gaussian random variable independent of $\mathcal{F}_{s}$, with distribution function $\phi(\cdot)$. We easily obtain

$$
\begin{aligned}
p_{1}\left(a_{i}, S_{s}^{i}\right)= & -\mathbb{E}_{s}\left[\mathbb{1}_{X \leq w} \phi\left(\frac{\ln \left(\frac{a_{i} \alpha}{S_{s}^{i}}\right)-\mu_{i} \delta-\sigma_{i} \sqrt{\delta-\frac{1}{2}} X}{\sigma_{i} \sqrt{1 / 2}}\right)\right]+\left(\frac{a_{i} \alpha}{S_{s}^{i}}\right)^{\frac{2 \mu_{i}}{\sigma_{i}^{2}}} e^{-\frac{2 \mu_{i}^{2}}{\sigma_{i}^{2}}\left(\delta-\frac{1}{2}\right)} \times \\
& \times \mathbb{E}_{s}\left[\mathbb{1}_{X \leq w} e^{-\frac{2 \mu_{i}}{\sigma_{i}} \sqrt{\delta-\frac{1}{2}} X} \phi\left(\frac{-\ln \left(\frac{a_{i} \alpha}{S_{s}^{i}}\right)+\mu_{i}(\delta-1)+\sigma_{i} \sqrt{\delta-\frac{1}{2}} X}{\sigma_{i} \sqrt{1 / 2}}\right)\right]
\end{aligned}
$$




$$
\begin{aligned}
p_{2}\left(a_{i}, S_{s}^{i}\right)= & 1-\mathbb{E}_{s}\left[\mathbb{1}_{X \leq w} \phi\left(\frac{\ln \left(\frac{a_{i}-x}{S_{s}^{i}}\right)-\mu_{i} \delta-\sigma_{i} \sqrt{\delta-\frac{1}{2}} X}{\sigma_{i} \sqrt{1 / 2}}\right)\right]+\left(\frac{a_{i} \alpha}{S_{s}^{i}}\right)^{\frac{2 \mu_{i}}{\sigma_{i}^{2}}} e^{-\frac{2 \mu_{i}^{2}}{\sigma_{i}^{2}}\left(\delta-\frac{1}{2}\right)} \times \\
& \times \mathbb{E}_{s}\left[\mathbb{1}_{X \leq w} e^{-\frac{2 \mu_{i}}{\sigma_{i}} \sqrt{\delta-\frac{1}{2}} X} \phi\left(\frac{-\ln \left(\frac{\left(a_{i}-x\right) S_{s}^{i}}{a^{2} \alpha^{2}}\right)+\mu_{i}(\delta-1)+\sigma_{i} \sqrt{\delta-\frac{1}{2}} X}{\sigma_{i} \sqrt{1 / 2}}\right)\right]
\end{aligned}
$$

This result could be enhanced as the expectations involved in the formulas of $p_{1}$ and $p_{2}$ can be explicitly computed using Equation (1.2).

A.2 Proof of Proposition 2. We remark that $\mathbb{P}_{s}\left(\Gamma_{t}>0\right)=1-\mathbb{P}_{s}\left(\Gamma_{t}=0\right)=1-\mathbb{P}_{s}\left(\tilde{S}_{t}>\right.$ $\left.\alpha \max \left(a_{1} \ldots a_{n}\right)\right)$ and we conclude as in the proof of Proposition 1 that $\mathbb{P}_{s}\left(\Gamma_{t}>0\right)=1-p_{1}\left(\max \left(a_{1} \ldots a_{n}\right), S_{s}\right)$, which is the desired result.

A.3 Proof of Proposition 3. We notice that the expected impaired amount for a single share $S$ with an acquisition cost $a_{i}$ can be computed as follows

$$
\mathbb{E}_{s}\left(P D D_{t}^{i}\right)=a_{i} \mathbb{P}_{s}\left(\widetilde{S}_{t} \leq a_{i} \alpha\right)-\mathbb{E}_{s}\left(S_{t} \mathbb{1}_{\widetilde{S}_{t} \leq a_{i} \alpha}\right) .
$$

The first term $\mathbb{P}_{s}\left(\widetilde{S}_{t} \leq a_{i} \alpha\right)=1-p_{1}\left(a_{i}, S_{s}\right)$. The second term can be expressed, using the Markov property, in the following form:

$$
\mathbb{E}_{s}\left[S_{t-\frac{1}{2}} \mathbb{1}_{S_{t-\frac{1}{2}} \leq a_{i} \alpha} \mathbb{E}_{t-\frac{1}{2}}\left(e^{\frac{\mu}{2}+\sigma B_{\frac{1}{2}}} \mathbb{1}_{\sup _{u \in\left[0, \frac{1}{2}\right]} \mu u+\sigma B_{u} \leq \ln a_{i} \alpha / S_{t-1 / 2}}\right)\right],
$$

which needs the density $\mathbb{P}\left(X_{t} \in d x, \sup _{u \in[0, t]} X_{u} \leq a\right)$. Now, using this density we can write that $\mathbb{E}_{t-\frac{1}{2}}(\ldots)$ equals

$$
e^{r / 2} \phi\left(\frac{\ln \frac{a_{i} \alpha}{S_{t-\frac{1}{2}}}-\frac{\mu+\sigma^{2}}{2}}{\sigma \sqrt{1 / 2}}\right)-\left(\frac{a_{i} \alpha}{S_{t-\frac{1}{2}}}\right)^{\frac{2\left(\mu+\sigma^{2}\right)}{\sigma^{2}}} e^{r / 2} \phi\left(\frac{-\ln \frac{a_{i} \alpha}{S_{t-\frac{1}{2}}}-\frac{\mu+\sigma^{2}}{2}}{\sigma \sqrt{1 / 2}}\right) .
$$

Finally, using the fact that $S_{t-\frac{1}{2}}=S_{s} e^{\mu\left(\delta-\frac{1}{2}\right)+\sigma \sqrt{\delta-\frac{1}{2}} X}$, with $\delta=t-s$ and $X$ a standard normal r.v. independent of $\mathcal{F}_{s}$, together with the identity in Equation (1.2) leads to the desired formula.

A.4 Proof of Theorem 1. To find the joint probability $\mathbb{P}_{t}\left(\Gamma_{t+1} \leq y, \Sigma_{t+1} \leq x\right)$, we will need the following technical lemma (to prove it, we use Equation (1.2)).

Lemma 1 Let $a>0, \beta, \gamma$ and $\alpha$ be some constants, then for $s, t \geq 0$ we have the following

$$
\begin{aligned}
\mathbb{E}_{t} & {\left[\mathbb{1}_{S_{t+s}<a} S_{t+s}^{-\gamma} \phi\left(\alpha+\beta \ln S_{t+s}\right)\right]=S_{t}^{-\gamma} e^{-\gamma\left(r-\frac{\sigma^{2}}{2}\right) s} \times } \\
& \Phi\left(\frac{\ln \frac{a}{S_{t}}-\left(r-\frac{\sigma^{2}}{2}-\gamma \sigma^{2}\right) s}{\sigma \sqrt{s}}, \frac{\alpha+\beta \ln \left(S_{t}\right)+\beta s\left(r-\frac{\sigma^{2}}{2}\right)-\beta \gamma \sigma^{2} s}{\sqrt{1+\beta^{2} \sigma^{2} s}} ;-\frac{\beta \sigma \sqrt{s}}{\sqrt{1+\beta^{2} \sigma^{2} s}}\right) .
\end{aligned}
$$

Similarly, we have

$$
\mathbb{E}_{t}\left[\mathbb{1}_{S_{t+s}>a} \phi\left(\alpha+\beta \ln S_{t+s}\right)\right]=\mathbb{E}_{t}\left[\phi\left(\alpha+\beta \ln S_{t+s}\right)\right]-\mathbb{E}_{t}\left[\mathbb{1}_{S_{t+s}<a} \phi\left(\alpha+\beta \ln S_{t+s}\right)\right]
$$




$$
\begin{aligned}
= & \Phi\left(0, \frac{\alpha+\beta \ln S_{t}+\beta s\left(r-\frac{\sigma^{2}}{2}\right)}{\sqrt{1+\beta^{2} \sigma^{2} s}} ;-\frac{\beta \sigma \sqrt{s}}{\sqrt{1+\beta^{2} \sigma^{2} s}}\right)+\Phi\left(0, \frac{\alpha+\beta \ln S_{t}+\beta s\left(r-\frac{\sigma^{2}}{2}\right)}{\sqrt{1+\beta^{2} \sigma^{2} s}} ; \frac{\beta \sigma \sqrt{s}}{\sqrt{1+\beta^{2} \sigma^{2} s}}\right) \\
& -\Phi\left(\frac{\ln \frac{a}{S_{t}}-\left(r-\frac{\sigma^{2}}{2}-\gamma \sigma^{2}\right) s}{\sigma \sqrt{s}}, \frac{\alpha+\beta \ln \left(S_{t}\right)+\beta s\left(r-\frac{\sigma^{2}}{2}\right)}{\sqrt{1+\beta^{2} \sigma^{2} s}} ;-\frac{\beta \sigma \sqrt{s}}{\sqrt{1+\beta^{2} \sigma^{2} s}}\right) .
\end{aligned}
$$

Let $n \geq 1$, we are concerned with the computation of the conditional joint c.d.f. of $\left(\Gamma_{t+1}, \Sigma_{t+1}\right)$ :

$$
\mathbb{P}_{t}\left(\Gamma_{t+1} \leq y, \Sigma_{t+1} \leq x\right)=\mathbb{E}_{t}\left[\mathbb{P}_{t+\frac{1}{2}}\left(S_{t+1} \leq \frac{x}{n}, \sum_{i=1}^{n}\left(a_{i}-S_{t+1}\right)^{+} \mathbb{1}_{\widetilde{S}_{t+1} \leq a_{i} \alpha} \leq y\right)\right]
$$

In the above equation, the probability conditional on $\mathcal{F}_{t+\frac{1}{2}}$ can be decomposed in the following form:

$$
\begin{aligned}
\mathbb{P}_{t+\frac{1}{2}}\left(S_{t+1} \leq \frac{x}{n}, \tilde{S}_{t+1} \leq a_{1} \alpha, \sum_{i=1}^{n}\left(a_{i}-S_{t+1}\right)^{+} \leq y\right) \\
+\cdots+\mathbb{P}_{t+\frac{1}{2}}\left(S_{t+1} \leq \frac{x}{n}, a_{j} \alpha<\widetilde{S}_{t+1} \leq a_{j+1} \alpha, \sum_{i=j+1}^{n}\left(a_{i}-S_{t+1}\right)^{+} \leq y\right) \\
+\cdots+\mathbb{P}_{t+\frac{1}{2}}\left(S_{t+1} \leq \frac{x}{n}, \widetilde{S}_{t+1} \geq a_{n} \alpha\right) .
\end{aligned}
$$

It is easily seen that the term (1.3) can be written as

$$
\mathbb{P}_{t+\frac{1}{2}}\left(\widetilde{S}_{t+1} \leq a_{1} \alpha, \frac{1}{n}\left(\sum_{i=1}^{n} a_{i}-y\right) \leq S_{t+1} \leq \frac{x}{n} \wedge a_{1} \alpha\right),
$$

and similarly the term (1.4) as

$$
\mathbb{P}_{t+\frac{1}{2}}\left(a_{j} \alpha<\widetilde{S}_{t+1} \leq a_{j+1} \alpha, \frac{1}{n-j}\left(\sum_{i=j+1}^{n} a_{i}-y\right) \leq S_{t+1} \leq \frac{x}{n} \wedge a_{j+1} \alpha\right)
$$

Moreover, we use Markov property and Proposition 6 to write the term (1.5) in the following form

$$
\begin{aligned}
& \mathbb{1}_{S_{t+\frac{1}{2}} \geq a_{n} \alpha} \phi\left(\frac{\ln \frac{x}{n S_{t+\frac{1}{2}}}-\frac{\mu}{2}}{\sigma \sqrt{0.5}}\right)+\mathbb{1}_{S_{t+\frac{1}{2}}<a_{n} \alpha} \mathbb{1}_{\frac{x}{n} \leq a_{n} \alpha}\left(\frac{a_{n} \alpha}{S_{t+\frac{1}{2}}}\right)^{\frac{2 \mu}{\sigma^{2}}} \phi\left(\frac{\ln \frac{x S_{t+\frac{1}{2}}}{n a_{n}^{2} \alpha^{2}}-\frac{\mu}{2}}{\sigma \sqrt{0.5}}\right)+ \\
& \mathbb{1}_{S_{t+\frac{1}{2}}<a_{n} \alpha} \mathbb{1}_{\frac{x}{n}>a_{n} \alpha}\left[\phi\left(\frac{\ln \frac{x}{n S_{t+\frac{1}{2}}}-\frac{\mu}{2}}{\sigma \sqrt{0.5}}\right)-\phi\left(\frac{\ln \frac{a_{n} \alpha}{S_{t+\frac{1}{2}}}-\frac{\mu}{2}}{\sigma \sqrt{0.5}}\right)+\left(\frac{a_{n} \alpha}{S_{t+\frac{1}{2}}}\right)^{\frac{2 \mu}{\sigma^{2}}} \phi\left(\frac{-\ln \frac{a_{n} \alpha}{S_{t+\frac{1}{2}}}-\frac{\mu}{2}}{\sigma \sqrt{0.5}}\right)\right] .
\end{aligned}
$$

On $\left\{\frac{1}{n}\left(\sum_{i=1}^{n} a_{i}-y\right) \leq \frac{x}{n} \wedge a_{1} \alpha\right\}$, the term (1.3) is equal to :

$$
\begin{aligned}
\mathbb{1}_{S_{t+\frac{1}{2}}<a_{1} \alpha} & {\left[\phi\left(\frac{\ln \frac{\frac{x}{n} \wedge a_{1} \alpha}{S_{t+\frac{1}{2}}}-\frac{\mu}{2}}{\sigma \sqrt{0.5}}\right)-\left(\frac{a_{1} \alpha}{S_{t+\frac{1}{2}}}\right)^{\frac{2 \mu}{\sigma^{2}}} \phi\left(\frac{\ln \frac{\frac{x}{n} \wedge a_{1} \alpha}{S_{t+\frac{1}{2}}}-2 \ln \frac{a_{1} \alpha}{S_{t+\frac{1}{2}}}-\frac{\mu}{2}}{\sigma \sqrt{0.5}}\right)\right.} \\
& \left.-\phi\left(\frac{\ln \frac{\sum_{i=1}^{n} a_{i}-y}{n S_{t+\frac{1}{2}}}-\frac{\mu}{2}}{\sigma \sqrt{0.5}}\right)+\left(\frac{a_{1} \alpha}{S_{t+\frac{1}{2}}}\right)^{\frac{2 \mu}{\sigma^{2}}} \phi\left(\frac{\ln \frac{\sum_{i=1}^{n} a_{i}-y}{n S_{t+\frac{1}{2}}}-2 \ln \frac{a_{1} \alpha}{S_{t+\frac{1}{2}}}-\frac{\mu}{2}}{\sigma \sqrt{0.5}}\right)\right] .
\end{aligned}
$$


On $\left\{\frac{1}{n-j}\left(\sum_{i=1+j}^{n} a_{i}-y\right) \leq \frac{x}{n} \wedge a_{j+1} \alpha\right\}$ the term (1.4) is equal to :

$$
\begin{aligned}
& \mathbb{1}_{S_{t+\frac{1}{2}} \leq a_{j+1} \alpha}\left[\phi\left(\frac{\ln \frac{\frac{x}{n} \wedge a_{j+1} \alpha}{S_{t+\frac{1}{2}}}-\frac{\mu}{2}}{\sigma \sqrt{0.5}}\right)-\left(\frac{a_{j+1} \alpha}{S_{t+\frac{1}{2}}}\right)^{\frac{2 \mu}{\sigma^{2}}} \phi\left(\frac{\ln \frac{\frac{x}{n} \wedge a_{j+1} \alpha}{S_{t+\frac{1}{2}}}-2 \ln \frac{a_{j+1} \alpha}{S_{t+\frac{1}{2}}}-\frac{\mu}{2}}{\sigma \sqrt{0.5}}\right)\right. \\
& \left.-\phi\left(\frac{\ln \frac{\sum_{i=j+1}^{n} a_{i}-y}{(n-j) S_{t+\frac{1}{2}}}-\frac{\mu}{2}}{\sigma \sqrt{0.5}}\right)+\left(\frac{a_{j+1} \alpha}{S_{t+\frac{1}{2}}}\right)^{\frac{2 \mu}{\sigma^{2}}} \phi\left(\frac{\ln \frac{\sum_{i=j+1}^{n} a_{i}-y}{(n-j) S_{t+\frac{1}{2}}}-2 \ln \frac{a_{j+1} \alpha}{S_{t+\frac{1}{2}}}-\frac{\mu}{2}}{\sigma \sqrt{0.5}}\right)\right] \\
& +\mathbb{1}_{S_{t+\frac{1}{2}} \leq a_{j} \alpha}\left(\mathbb{1}_{\frac{1}{n-j}\left(\sum_{i=j+1}^{n} a_{i}-y\right)>a_{j} \alpha}-\mathbb{1}_{\frac{x}{n}>a_{j} \alpha}\right)\left[\phi\left(\frac{\ln \frac{a_{j} \alpha}{S_{t+\frac{1}{2}}}-\frac{\mu}{2}}{\sigma \sqrt{0.5}}\right)+\left(\frac{a_{j} \alpha}{S_{t+\frac{1}{2}}}\right)^{\frac{2 \mu}{\sigma^{2}}} \phi\left(\frac{\ln \frac{-a_{j} \alpha}{S_{t+\frac{1}{2}}}-\frac{\mu}{2}}{\sigma \sqrt{0.5}}\right)\right] \\
& +\mathbb{1}_{S_{t+\frac{1}{2}} \leq a_{j} \alpha} \mathbb{1}_{\frac{x}{n}<a_{j} \alpha}\left[\phi\left(\frac{\ln \frac{\frac{x}{n} \wedge a_{j+1} \alpha}{S_{t+\frac{1}{2}}}-\frac{\mu}{2}}{\sigma \sqrt{0.5}}\right)-\left(\frac{a_{j} \alpha}{S_{t+\frac{1}{2}}}\right)^{\frac{2 \mu}{\sigma^{2}}} \phi\left(\frac{\ln \frac{\frac{x}{n} \wedge a_{j+1} \alpha}{S_{t+\frac{1}{2}}}-2 \ln \frac{a_{j} \alpha}{S_{t+\frac{1}{2}}}-\frac{\mu}{2}}{\sigma \sqrt{0.5}}\right)\right] \\
& +\mathbb{1}_{S_{t+\frac{1}{2}} \leq a_{j} \alpha} \mathbb{1}_{\frac{1}{n-j}\left(\sum_{i=j+1}^{n} a_{i}-y\right) \leq a_{j} \alpha}\left[\phi\left(\frac{\ln \frac{\sum_{i=j+1}^{n} a_{i}-y}{(n-j) S_{t+\frac{1}{2}}}-\frac{\mu}{2}}{\sigma \sqrt{0.5}}\right)+\left(\frac{a_{j} \alpha}{S_{t+\frac{1}{2}}}\right)^{\frac{2 \mu}{\sigma^{2}}} \phi\left(\frac{\ln \frac{\sum_{i=j+1}^{n} a_{i}-y}{(n-j) S_{t+\frac{1}{2}}}-2 \ln \frac{a_{j} \alpha}{S_{t+\frac{1}{2}}}-\frac{\mu}{2}}{\sigma \sqrt{0.5}}\right)\right] .
\end{aligned}
$$

We conclude the proof using Lemma 1 to compute the expectations conditional to $\mathcal{F}_{t}$.

\section{B Correlated securities}

In order to prove our results in the correlated case, we shall need the joint distribution of two correlated Brownian motions with drift and their suprema. The joint distribution is obtain by making the following change of variables $x_{2}=M_{2}-\sigma_{2} r \sin \theta$ and $x_{1}=M_{1}-\sigma_{1} r\left(\rho \sin \theta+\sqrt{1-\rho^{2}} \cos \theta\right)$ in Lemma 3 and Remark 4 from He et al. (1998).

Proposition 8 Let $X^{i}=\mu_{i} t+\sigma_{i} W_{t}^{i}$, where $\mu_{i} \in \mathbb{R}, \sigma_{i}>0$ and $W^{i}$ two correlated Brownian motions with correlation coefficient $\rho \in]-1,1\left[\right.$. For $x_{1} \leq M_{1}, x_{2} \leq M_{2}$ with $M_{1}, M_{2} \geq 0$, we have :

$\mathbb{P}\left(X_{t}^{1} \in d x_{1}, X_{t}^{2} \in d x_{2}, \sup _{u \leq t} X_{u}^{1} \leq M_{1}, \sup _{u \leq t} X_{u}^{2} \leq M_{2}\right)=p\left(r, r_{0}, \theta, \theta_{0}, t ; M_{1}, M_{2}\right) d r d \theta$

$\mathbb{P}\left(\tilde{X}^{1}{ }_{0 \rightarrow t} \leq M_{1}, \tilde{X}^{2}{ }_{0 \rightarrow t} \leq M_{2}\right)=q\left(r_{0}, \theta_{0}, t ; M_{1}, M_{2}\right)$,

where $p$ and $q$ are given in Definition 1 and

$$
\begin{aligned}
& r=\frac{1}{\sqrt{1-\rho^{2}}} \sqrt{\frac{\left(M_{1}-x_{1}\right)^{2}}{\sigma_{1}^{2}}-2 \rho \frac{\left(M_{1}-x_{1}\right)\left(M_{2}-x_{2}\right)}{\sigma_{1} \sigma_{2}}+\frac{\left(M_{2}-x_{2}\right)^{2}}{\sigma_{2}^{2}}}, \\
& r_{0}=\frac{1}{\sqrt{1-\rho^{2}}} \sqrt{\frac{M_{1}^{2}}{\sigma_{1}^{2}}-2 \rho \frac{M_{1} M_{2}}{\sigma_{1} \sigma_{2}}+\frac{M_{2}^{2}}{\sigma_{2}^{2}}}, \quad \tan \beta=-\frac{\sqrt{1-\rho^{2}}}{\rho}, \quad \beta \in[0, \pi], \\
& \tan \theta=\sqrt{1-\rho^{2}}\left(\frac{\sigma_{2}\left(M_{1}-x_{1}\right)}{\sigma_{1}\left(M_{2}-x_{2}\right)}-\rho\right)^{-1}, \quad \tan \theta_{0}=\sqrt{1-\rho^{2}}\left(\frac{\sigma_{2} M_{1}}{\sigma_{1} M_{2}}-\rho\right)^{-1}, \quad \theta, \theta_{0} \in[0, \beta] .
\end{aligned}
$$

For some special correlation values, the function $q$ may be written in a simpler form (see Corollary 2 in He et al. (1998)). We easily obtain the following results ${ }^{4}$ :

\footnotetext{
${ }^{4}$ Let's note that there is a mistake in Corollary 2 He et al. (1998) and $t$ is sometimes missing
} 
Lemma 2 Under the assumptions of Proposition 8, if $\rho_{n}=-\cos \frac{\pi}{n}$, we have :

$$
\begin{aligned}
& p\left(r, r_{0}, \theta, \theta_{0}, t ; M_{1}, M_{2}\right) d r d \theta \mathbb{1}_{r \geq 0, \theta \in[0, \beta]}=p\left(x_{1}, x_{2}, t ; M_{1}, M_{2}\right) d x_{1} d x_{2} \mathbb{1}_{x_{1} \leq M_{1}, x_{2} \leq M_{2}}, \\
& q\left(r_{0}, \theta_{0}, t ; M_{1}, M_{2}\right)=q\left(M_{1}, M_{2}, t\right), \\
& \text { where } p\left(x_{1}, x_{2}, t ; M_{1}, M_{2}\right)=e^{\alpha_{1} x_{1}+\alpha_{2} x_{2}+b t} \sum_{k=1}^{n-1}\left[g_{k}^{+}\left(x_{1}, x_{2}, t\right)+g_{k}^{-}\left(x_{1}, x_{2}, t\right)\right] \text {, } \\
& q\left(M_{1}, M_{2}, t\right)=\sum_{k=0}^{n-1}\left[Q^{+}\left(M_{1}, M_{2}, \frac{2 k \pi}{n}+\theta\right)-Q^{-}\left(M_{1}, M_{2}, \frac{2 k \pi}{n}-\theta\right)\right], \\
& g_{k}^{+}\left(x_{1}, x_{2}, t\right)={ }_{-}^{+} \frac{1}{2 \pi t} \exp \left\{-\frac{1}{2 t}\left[\left(\frac{1}{\sqrt{1-\rho_{n}^{2}}}\left(\frac{M_{1}-x_{1}}{\sigma_{1}}-\rho_{n} \frac{M_{2}-x_{2}}{\sigma_{2}}\right)+\xi_{1}\right)^{2}+\left(\frac{M_{2}-x_{2}}{\sigma_{2}}-\xi_{2}\right)^{2}\right]\right\} \\
& Q^{+}\left(M_{1}, M_{2}, \frac{2 k \pi}{n}{ }_{-}^{+} \theta\right)=\exp \left[B_{0}+B_{1} \xi_{1}+B_{2} \xi_{2}+\left(\frac{B_{1}^{2}+B_{2}^{2}}{2}+b\right) t\right] \times \\
& \times \Phi\left(\frac{\xi_{2}+B_{2} t}{\sqrt{t}}, \frac{-\sqrt{1-\rho_{n}^{2}}\left(\xi_{1}+B_{1} t\right)+\rho_{n}\left(\xi_{2}+B_{2} t\right)}{\sqrt{t}}, \rho_{n}\right), \\
& \xi_{1}=-r_{0} \cos \left(\frac{2 k \pi}{n}{ }_{-}+\theta\right), \quad \xi_{2}=r_{0} \sin \left(\frac{2 k \pi}{n}{ }_{-}^{+} \theta\right) \\
& \tan \theta=-\frac{\sqrt{1-\rho_{n}^{2}}}{\rho_{n}-\frac{M_{1} \sigma_{2}}{M_{2} \sigma_{1}}}, \quad r_{0}=\frac{1}{\sqrt{1-\rho_{n}^{2}}} \sqrt{\frac{M_{1}^{2}}{\sigma_{1}^{2}}-2 \rho_{n} \frac{M_{1} M_{2}}{\sigma_{1} \sigma_{2}}+\frac{M_{2}^{2}}{\sigma_{2}^{2}}}, \\
& B_{0}=a_{1} M_{1}+a_{2} M_{2}, \quad B_{1}=a_{1} \sigma_{1} \sqrt{1-\rho_{n}^{2}}, \quad B_{2}=-\left(a_{1} \sigma_{1} \rho_{n}+a_{2} \sigma_{2}\right)=\frac{-\alpha_{2}}{\sigma_{2}}, \\
& b=-\frac{\alpha_{1}^{2} \sigma_{2}^{2}+\alpha_{2}^{2} \sigma_{1}^{2}}{2\left(1-\rho_{n}^{2}\right) \sigma_{1}^{2} \sigma_{2}^{2}}+\frac{\rho_{n} \alpha_{1} \alpha_{2}}{\left(1-\rho_{n}^{2}\right) \sigma_{1} \sigma_{2}}, \quad a_{1}=\frac{\alpha_{1} \sigma_{2}-\rho_{n} \alpha_{2} \sigma_{1}}{\left(1-\rho_{n}^{2}\right) \sigma_{1}^{2} \sigma_{2}}, \quad a_{2}=\frac{\alpha_{2} \sigma_{1}-\rho_{n} \alpha_{1} \sigma_{2}}{\left(1-\rho_{n}^{2}\right) \sigma_{1} \sigma_{2}^{2}} .
\end{aligned}
$$

B.1 Proof of Proposition 5. We remark that

$$
\begin{aligned}
\mathbb{P}_{s}\left(\Gamma_{t}>0\right) & =1-\mathbb{P}_{s}\left(\Gamma_{t}=0\right)=\mathbb{P}_{s}\left(\tilde{S}_{t}^{1} \leq \alpha a_{1}\right)+\mathbb{P}_{s}\left(\tilde{S}_{t}^{2} \leq \alpha a_{2}\right)-\mathbb{P}_{s}\left(\tilde{S}_{t}^{1} \leq \alpha a_{1}, \tilde{S}_{t}^{2} \leq \alpha a_{2}\right) \\
& =\sum_{i=1}^{2} p_{i}\left(a_{i}, S_{s}^{i}\right)-\mathbb{P}_{s}\left(\mathbb{P}_{t-1 / 2}\left(\tilde{S}_{t}^{1} \leq \alpha a_{1}, \tilde{S}_{t}^{2} \leq \alpha a_{2}\right)\right)
\end{aligned}
$$

Markov property, Proposition 8 and writing $S_{t-1 / 2}^{1}=S_{s}^{1} e^{\mu_{1}(\delta-1 / 2)+\sigma_{1} \sqrt{\delta-1 / 2} X}$ with $X \sim \mathcal{N}(0,1)$ lead to the desired formula.

We need some technical results in order to find the joint law of $\Gamma$ and $\Sigma$.

Lemma 3 Let $x>0$. The c.d.f of the aggregate dynamics $\Sigma$ of two correlated shares is given by

$$
\mathbb{P}_{t}\left(\Sigma_{t+1} \leq x\right)=\mathbb{E}\left[\mathbb{1}_{X \leq \frac{\ln \frac{x}{s_{1}}-\mu_{1}}{\sigma_{1}}} \phi\left(\frac{1}{\sigma_{2} \sqrt{1-\rho^{2}}} \ln \frac{x-s_{1} e^{\mu_{1}+\sigma_{1} X}}{s_{2} e^{\mu_{2}+\sigma_{2} \rho X}}\right)\right]_{s_{1}=S_{t}^{1}, s_{2}=S_{t}^{2}},
$$

where $X$ is a standard Gaussian r.v. We denote this probability by $f\left(S_{t}^{1}, S_{t}^{2}, x\right)=\mathbb{P}_{t}\left(\Sigma_{t+1} \geq 0\right)$. 
Proof. Since $W^{1}$ and $W^{2}$ are two correlated Brownian motions with correlation coefficient $\rho$, then $W^{2}=$ $\rho W^{1}+\sqrt{1-\rho^{2}} W$ with $W^{1}$ and $W$ two independent BM. Hence :

$$
\mathbb{P}_{t}\left(\Sigma_{t+1} \leq 0\right)=\mathbb{P}_{t}\left(S_{t}^{1} e^{\mu_{1}+\sigma_{1} X}+S_{t}^{2} e^{\mu_{2}+\sigma_{2} \rho X+\sigma_{2} \sqrt{1-\rho^{2}} Z} \leq x\right),
$$

where $X$ and $Z$ are two independent standard Gaussian r.v. We integrate with respect $Z$ and the conclusion holds.

Lemma 4 Let $X_{t}^{i}=\mu_{i} t+\sigma_{i} W_{t}^{i}$, where $\mu_{i}, \sigma_{i} \in \mathbb{R}$ and $W^{i}$ two correlated BM with correlation coefficient $\rho \in]-1,1\left[\right.$. Then for $M_{1}>0, x_{1}, x_{2} \in \mathbb{R}$

$$
\mathbb{P}\left(X_{t}^{1} \in d x_{1}, X_{t}^{2} \in d x_{2}, \sup _{u \leq t} X_{u}^{1} \leq M_{1}\right)=h\left(x_{1}, x_{2}, M_{1}\right) d x_{1} d x_{2}
$$

where

$h\left(x_{1}, x_{2}, M_{1}\right)=\mathbb{1}_{x_{1}<M_{1}}\left[e^{-\frac{\left(x_{1}-\mu_{1} t\right)^{2}}{2 \sigma_{1}^{2} t}}-e^{2 \frac{\mu_{1} M_{1}}{\sigma_{1}^{2}}} e^{-\frac{\left(x_{1}-2 M_{1}-\mu_{1} t\right)^{2}}{2 \sigma_{1}^{2} t}}\right] \frac{1}{2 \pi t \sigma_{1} \sigma_{2} \sqrt{1-\rho^{2}}} e^{-\frac{\left(\sigma_{1} x_{2}-\rho \sigma_{2} x_{1}+\left(\mu_{1} \sigma_{2} \rho-\mu_{2} \sigma_{1}\right) t\right)^{2}}{2 \sigma_{1}^{2} \sigma_{2}^{2} t\left(1-\rho^{2}\right)}}$.

Proof. Without loss of generality we consider $\sigma_{i}=1, i=1,2$ (we then replace $x_{1}, M_{1}, x_{2}$ by $\frac{x_{1}}{\sigma_{1}}, \frac{M_{1}}{\sigma_{1}}, \frac{x_{2}}{\sigma_{2}}$ ). Since

$$
\mathbb{P}\left(X_{t}^{1} \in d x_{1}, X_{t}^{2} \in d x_{2}, \sup _{u \leq t} X_{u}^{1} \leq M_{1}\right)=\frac{\partial^{2}}{\partial x_{1} \partial x_{2}} \mathbb{P}\left(X_{t}^{1} \leq x_{1}, X_{t}^{2} \leq x_{2}, \sup _{u \leq t} X_{u}^{1} \leq M_{1}\right)
$$

and $W^{2}=\rho W^{1}+\sqrt{1-\rho^{2}} W$ with $W^{1}$ and $W$ two independent BM, we can write for $\rho \neq 0^{5}$

$$
\begin{aligned}
& \mathbb{P}\left(X_{t}^{1} \leq x_{1}, X_{t}^{2} \leq x_{2}, \sup _{u \leq t} X_{u}^{1} \leq M_{1}\right) \\
& =\mathbb{1}_{x_{1} \geq M_{1}} \mathbb{P}\left(X_{t}^{2} \leq x_{2}, \sup _{u \leq t} X_{u}^{1} \leq M_{1}\right)+\mathbb{1}_{x_{1}<M_{1}} \mathbb{P}\left(X_{t}^{1} \leq x_{1} \wedge \frac{1}{\rho}\left(x_{2}-\sqrt{1-\rho^{2}} W_{t}+\left(\mu_{1} \rho-\mu_{2}\right) t\right), \text { sup }_{u \leq t} X_{u}^{1} \leq M_{1}\right) .
\end{aligned}
$$

The derivative of the first term is 0 . For the second term, we integrate with respect to the law of $W_{t}$ and then we use Proposition 6. Finally the derivative with respect to $x_{1}$ and $x_{2}$ leads to the desired result.

We easily deduce the following :

Corollary 4 Under the assumptions of Lemma 4 let $R_{1}, R_{2}, x, w, M_{1}>0$. Then

$$
\begin{aligned}
& \mathbb{P}\left(R_{1} e^{X_{t}^{1}}+R_{2} e^{X_{t}^{2}} \leq x, \sup _{u \leq t} X_{u}^{1} \leq M_{1}\right)=\mathbb{E}\left[\mathbb{1}_{X \leq \frac{\left(\ln \left(\frac{x}{R_{1}}\right) \wedge M_{1}\right)-\mu_{1} t}{\sigma_{1} \sqrt{t}}} \phi\left(\frac{1}{\sigma_{2} \sqrt{t\left(1-\rho^{2}\right)}} \ln \frac{x-R_{1} e^{\sigma_{1} X \sqrt{t}+\mu_{1} t}}{R_{2} e^{X \rho \sigma_{2} \sqrt{t}+\mu_{2} t}}\right)\right] \\
& \left.-e^{2 \frac{\mu_{1} M_{1}}{\sigma_{1}^{2}}} \mathbb{E}\left[\begin{array}{l}
\mathbb{1} \quad \\
X \leq \frac{\left(\ln \left(\frac{x}{R_{1}}\right) \wedge M_{1}\right)-2 M_{1}-\mu_{1} t}{\sigma_{1} \sqrt{t}}
\end{array} \mid \frac{1}{\sigma_{2} \sqrt{t\left(1-\rho^{2}\right)}} \ln \frac{x-R_{1} e^{\sigma_{1} X \sqrt{t}+2 M_{1}+\mu_{1} t}}{R_{2} e^{X \sigma_{2} \sqrt{t}+2 \frac{\sigma_{2}}{\sigma_{1}} \rho M_{1}+\mu_{2} t}}\right)\right],
\end{aligned}
$$

$$
\begin{aligned}
& { }^{5} \text { If } \rho=0 \text {, then } \\
& \begin{aligned}
\mathbb{P}\left(X_{t}^{1} \in d x_{1}, X_{t}^{2} \in d x_{2}, \sup _{u \leq t} X_{u}^{1} \leq M_{1}\right) & =\mathbb{P}\left(X_{t}^{1} \in d x_{1}, \sup _{u \leq t} X_{u}^{1} \leq M_{1}\right) \mathbb{P}\left(X_{t}^{2} \in d x_{2}\right) \\
& =\mathbb{1}_{x_{1}<M_{1}}\left[e^{-\frac{\left(x_{1}-\mu_{1} t\right)^{2}}{2 t}}-e^{2 \mu_{1} M_{1}} e^{-\frac{\left(x_{1}-2 M_{1}-\mu_{1} t\right)^{2}}{2 t}}\right] \frac{1}{2 \pi t} e^{-\frac{\left(x_{2}-\mu_{2} t\right)^{2}}{2 t}} d x_{1} d x_{2}
\end{aligned}
\end{aligned}
$$




$$
\begin{aligned}
& \mathbb{P}\left(R_{1} e^{X_{t}^{1}}+R_{2} e^{X_{t}^{2}} \leq x, \sup _{u \leq t} X_{u}^{1} \leq M_{1}, e^{X_{t}^{1}} \geq w\right)=\mathbb{1}_{w \leq 0} \mathbb{P}\left(R_{1} e^{X_{t}^{1}}+R_{2} e^{X_{t}^{2}} \leq x, \sup _{u \leq t} X_{u}^{1} \leq M_{1}\right) \\
& +\mathbb{1}_{0<w \leq \frac{x}{R_{1}} \wedge e^{M_{1}}}\left[\mathbb{E}\left(\mathbb{1}_{\frac{\ln w-\mu_{1} t}{\sigma_{1} \sqrt{t}} \leq X \leq \frac{\left(\ln \left(\frac{x}{R_{1}}\right) \wedge M_{1}\right)-\mu_{1} t}{\sigma_{1} \sqrt{t}}} \phi\left(\frac{1}{\sigma_{2} \sqrt{t\left(1-\rho^{2}\right)}} \ln \frac{x-R_{1} e^{\sigma_{1} X \sqrt{t}+\mu_{1} t}}{R_{2} e^{X \sigma_{2} \rho \sqrt{t}+\mu_{2} t}}\right)\right)\right. \\
& \left.-e^{2 \frac{\mu_{1} M_{1}}{\sigma_{1}^{2}}} \mathbb{E}\left(\mathbb{1} \underset{\frac{\ln w-\mu_{1} t-2 M_{1}}{\sigma_{1} \sqrt{t}} \leq X \leq}{\frac{\left(\ln \left(\frac{x}{R_{1}}\right) \wedge M_{1}\right)-2 M_{1}-\mu_{1} t}{\sigma_{1} \sqrt{t}}} \phi\left(\frac{1}{\sigma_{2} \sqrt{t\left(1-\rho^{2}\right)}} \ln \frac{x-R_{1} e^{\sigma_{1} X \sqrt{t}+2 M_{1}+\mu_{1} t}}{R_{2} e^{X \sigma_{2} \rho \sqrt{t}+2 \rho \frac{\sigma_{2}}{\sigma_{1}} M_{1}+\mu_{2} t}}\right)\right)\right],
\end{aligned}
$$

where $X$ is a standard Gaussian r.v.

B.2 Proof of Theorem 2. The joint c.d.f

$$
\mathbb{P}_{t}\left(\Gamma_{t+1} \leq y, \Sigma_{t+1} \leq x\right)=\mathbb{P}_{t}\left(\left(a_{1}-S_{t+1}^{1}\right) \mathbb{1}_{\tilde{S}_{t+1}^{1} \leq \alpha a_{1}}+\left(a_{2}-S_{t+1}^{2}\right) \mathbb{1}_{\tilde{S}_{t+1}^{2} \leq \alpha a_{2}} \leq y, S_{t+1}^{1}+S_{t+1}^{2} \leq x\right),
$$

can be decomposed in the following form :

$$
\begin{aligned}
& \mathbb{P}_{t}\left(S_{t+1}^{1}+S_{t+1}^{2} \leq x, \tilde{S}_{t+1}>\alpha a_{1}, \tilde{S}^{2}{ }_{t+1}>\alpha a_{2}\right) \\
& +\sum_{i, j \in\{1,2\}, i \neq j} \mathbb{P}_{t}\left(S_{t+1}^{1}+S_{t+1}^{2} \leq x, \tilde{S}^{i} t+1 \leq \alpha a_{i}, \tilde{S}^{j} t+1>\alpha a_{j}, S_{t+1}^{i} \geq a_{i}-y\right) \\
& +\mathbb{P}_{t}\left(a_{1}+a_{2}-y \leq S_{t+1}^{1}+S_{t+1}^{2} \leq x, \tilde{S}_{t+1} \leq \alpha a_{1}, \tilde{S}_{t+1}^{2} \leq \alpha a_{2}\right) .
\end{aligned}
$$

\section{Step 1 - Probabilities decomposition}

- The first term $C_{1}\left(S_{t}^{1}, S_{t}^{2}\right)=\mathbb{P}_{t}\left(S_{t+1}^{1}+S_{t+1}^{2} \leq x, \tilde{S}^{1}{ }_{t+1}>\alpha a_{1}, \tilde{S}_{t+1}>\alpha a_{2}\right)$ may be written as :

$$
\begin{aligned}
\mathbb{1}_{x \geq \alpha\left(a_{1}+a_{2}\right)} & {\left[\mathbb{P}_{t}\left(S_{t+1}^{1}+S_{t+1}^{2} \leq x\right)-\mathbb{P}_{t}\left(S_{t+1}^{1}+S_{t+1}^{2} \leq x, \tilde{S}_{t+1} \leq \alpha a_{1}\right)\right.} \\
- & \left.\mathbb{P}_{t}\left(S_{t+1}^{1}+S_{t+1}^{2} \leq x, \tilde{S}^{2}{ }_{t+1} \leq \alpha a_{2}\right)+\mathbb{P}_{t}\left(S_{t+1}^{1}+S_{t+1}^{2} \leq x, \tilde{S}^{1}{ }_{t+1} \leq \alpha a_{1}, \tilde{S}^{2}{ }_{t+1} \leq \alpha a_{2}\right)\right]
\end{aligned}
$$

Recall that $S_{t+1}^{1}+S_{t+1}^{2}=\Sigma_{t+1}$. Note that on $\left\{x \geq \alpha\left(a_{1}+a_{2}\right)\right\}$,

$$
\mathbb{P}_{t}\left(S_{t+1}^{1}+S_{t+1}^{2} \leq x, \tilde{S}_{t+1} \leq \alpha a_{1}, \tilde{S}_{t+1}^{2} \leq \alpha a_{2}\right)=\mathbb{P}_{t}\left(\tilde{S}_{t+1}^{1} \leq \alpha a_{1}, \tilde{S}_{t+1}^{2} \leq \alpha a_{2}\right)
$$

Hence,

$$
\begin{aligned}
C_{1}\left(S_{t}^{1}, S_{t}^{2}\right)=\mathbb{1}_{x \geq \alpha\left(a_{1}+a_{2}\right)} & {\left[\mathbb{P}_{t}\left(\Sigma_{t+1} \leq x\right)-\mathbb{P}_{t}\left(\Sigma_{t+1} \leq x, \tilde{S}_{t+1} \leq \alpha a_{1}\right)\right.} \\
- & \left.\mathbb{P}_{t}\left(\Sigma_{t+1} \leq x, \tilde{S}^{2}{ }_{t+1} \leq \alpha a_{2}\right)+\mathbb{P}_{t}\left(\tilde{S}^{1}{ }_{t+1} \leq \alpha a_{1}, \tilde{S}^{2}{ }_{t+1} \leq \alpha a_{2}\right)\right] .
\end{aligned}
$$

- The second term $C_{2}\left(S_{t}^{i}, S_{t}^{j}, a_{i}, a_{j}\right)=\mathbb{P}_{t}\left(S_{t+1}^{1}+S_{t+1}^{2} \leq x, \tilde{S}^{i} t+1 \leq \alpha a_{i}, \tilde{S}^{j}{ }_{t+1}>\alpha a_{j}, S_{t+1}^{i} \geq a_{i}-y\right)$ equals to :

$$
\begin{aligned}
& \mathbb{1}_{y \geq a_{i}} \mathbb{1}_{\alpha a_{j} \leq x<\alpha\left(a_{1}+a_{2}\right)}\left[\mathbb{P}_{t}\left(\Sigma_{t+1} \leq x\right)-\mathbb{P}_{t}\left(\Sigma_{t+1} \leq x, \tilde{S}^{j} t+1 \leq \alpha a_{j}\right)\right] \\
+ & \mathbb{1}_{y \geq a_{i}} \mathbb{1}_{x \geq \alpha\left(a_{1}+a_{2}\right)}\left[\mathbb{P}_{t}\left(\Sigma_{t+1} \leq x, \tilde{S}_{t+1}^{i} \leq \alpha a_{i}\right)-\mathbb{P}_{t}\left(\tilde{S}^{i}{ }_{t+1} \leq \alpha a_{i}, \tilde{S}^{j} t+1 \leq \alpha a_{j}\right)\right]
\end{aligned}
$$




$$
\begin{aligned}
& +\quad \mathbb{1}_{a_{i}(1-\alpha) \leq y<a_{i}} \mathbb{1}_{x \geq\left(\alpha a_{j}\right) \vee\left(a_{i}-y\right)}\left[\mathbb{P}_{t}\left(\Sigma_{t+1} \leq x, \tilde{S}^{i}{ }_{t+1} \leq \alpha a_{i}, S_{t+1}^{i} \geq a_{i}-y\right)\right. \\
& \left.-\quad \mathbb{P}_{t}\left(\Sigma_{t+1} \leq x, \tilde{S}_{t+1}^{i} \leq \alpha a_{i}, \tilde{S}^{j}{ }_{t+1} \leq \alpha a_{j}, S_{t+1}^{i} \geq a_{i}-y\right)\right] .
\end{aligned}
$$

- The last term $C_{3}\left(S_{t}^{1}, S_{t}^{2}\right)=\mathbb{P}_{t}\left(a_{1}+a_{2}-y \leq S_{t+1}^{1}+S_{t+1}^{2} \leq x, \tilde{S}_{t+1} \leq \alpha a_{1}, \tilde{S}^{2}{ }_{t+1} \leq \alpha a_{2}\right)$ may be written as:

$$
\begin{aligned}
& \mathbb{1}_{x \geq \alpha\left(a_{1}+a_{2}\right)} \mathbb{1}_{y \geq a_{1}+a_{2}} \mathbb{P}_{t}\left(\tilde{S}_{t+1} \leq \alpha a_{1}, \tilde{S}^{2} t+1\right. \\
+ & \mathbb{1}_{x \geq\left(a_{1}+a_{2}-y\right) \vee \alpha\left(a_{1}+a_{2}\right)} \mathbb{1}_{y<a_{1}+a_{2}} \mathbb{P}_{t}\left(\Sigma_{t+1} \geq a_{1}+a_{2}-y, \tilde{S}^{1}{ }_{t+1} \leq \alpha a_{1}, \tilde{S}^{2}{ }_{t+1} \leq \alpha a_{2}\right) \\
+ & \mathbb{1}_{x<\alpha\left(a_{1}+a_{2}\right)} \mathbb{1}_{y \geq a_{1}+a_{2}} \mathbb{P}_{t}\left(\Sigma_{t+1} \leq x, \tilde{S^{1}} t+1 \leq \alpha a_{1}, \tilde{S^{2}} t+1 \leq \alpha a_{2}\right) \\
+ & \mathbb{1}_{0<a_{1}+a_{2}-y \leq x<\alpha\left(a_{1}+a_{2}\right)} \mathbb{P}_{t}\left(a_{1}+a_{2}-y \leq \Sigma_{t+1} \leq x, \tilde{S}_{t+1} \leq \alpha a_{1}, \tilde{S}^{2}{ }_{t+1} \leq \alpha a_{2}\right) .
\end{aligned}
$$

\section{Step 2 - Grouping of terms}

Remark that for the term $C_{2}\left(S_{t}^{i}, S_{t}^{j}, a_{i}, a_{j}\right)$, we may use Markov property and Proposition 8. Hence we can write (consider for example the case $i=1$ and $j=2$ ) :

$$
\begin{aligned}
& \mathbb{P}_{t}\left(\Sigma_{t+1} \leq x, \tilde{S}^{1}{ }_{t+1} \leq \alpha a_{1}, \tilde{S}^{2}{ }_{t+1} \leq \alpha a_{2}, S_{t+1}^{1} \geq a_{1}-y\right) \\
= & \mathbb{E}_{t}\left[\mathbb{1}_{S_{t+\frac{1}{2}}^{1} \leq \alpha a_{1}, S_{t+\frac{1}{2}}^{2} \leq \alpha a_{2}} \mathbb{P}_{t+\frac{1}{2}}\left(S_{t+\frac{1}{2}}^{1} e^{X_{\frac{1}{2}}^{1}}+S_{t+\frac{1}{2}}^{2} e^{X_{\frac{1}{2}}^{2}} \leq x, \tilde{X}_{\frac{1}{2}}^{1} \leq \ln \frac{\alpha a_{1}}{S_{t+\frac{1}{2}}^{1}}, \tilde{X}_{\frac{1}{2}}^{2} \leq \ln \frac{\alpha a_{2}}{S_{t+\frac{1}{2}}^{2}}, X_{\frac{1}{2}}^{1} \geq \ln \frac{a_{1}-y}{S_{t+\frac{1}{2}}^{1}}\right)\right], \\
= & \int_{0}^{\infty} \int_{0}^{\beta} \mathbb{E}_{t}\left[\mathbb{1}_{X \leq A_{1}, Y \leq A_{2}} p\left(\tilde{r}, \tilde{\theta}, \sigma_{1} \sqrt{0.5}\left(A_{1}-X\right), \sigma_{2} \sqrt{0.5}\left(A_{2}-Y\right)\right)\right]_{s_{i}=S_{t}^{i}} \mathbb{1}_{(\tilde{r}, \tilde{\theta}) \in D_{1}} d \tilde{r} d \tilde{\theta},
\end{aligned}
$$

where $X_{t}^{i}=\mu_{i} t+\sigma_{i} B_{t}^{i}$ where $B^{i}$ are two correlated BM independent of $\mathcal{F}_{t+\frac{1}{2}}$, and $D_{1}$ given before.

The same reasoning allows to write $\mathbb{P}_{t}\left(\Sigma_{t+1} \leq x, \tilde{S}^{1} t+1 \leq \alpha a_{1}, \tilde{S}_{t+1} \leq \alpha a_{2}, S_{t+1}^{2} \geq a_{2}-y\right), \mathbb{P}_{t}\left(\Sigma_{t+1} \geq\right.$ $\left.a_{1}+a_{2}-y, \tilde{S}^{1}{ }_{t+1} \leq \alpha a_{1}, \tilde{S}^{2}{ }_{t+1} \leq \alpha a_{2}\right), \mathbb{P}_{t}\left(\Sigma_{t+1} \leq x, \tilde{S}_{t+1} \leq \alpha a_{1}, \tilde{S}^{2}{ }_{t+1} \leq \alpha a_{2}\right)$ and $\mathbb{P}_{t}\left(a_{1}+a_{2}-y \leq \Sigma_{t+1} \leq\right.$ $\left.x, \tilde{S}_{t+1}^{1} \leq \alpha a_{1}, \tilde{S}_{t+1}^{2} \leq \alpha a_{2}\right)$ in a similar form with $D_{2}, D_{3}\left(a_{1}+a_{2}-y\right), D_{3}(x)$ and $D_{3}(x)-D_{3}\left(a_{1}+a_{2}-y\right)$. Hence, we have

$$
\begin{aligned}
& \mathbb{P}_{t}\left(\Gamma_{t+1} \leq y, \Sigma_{t+1} \leq x\right)=\sum_{i, j \in\{1,2\}, i \neq j} \mathbb{P}_{t}\left(\Sigma_{t+1} \leq x, \tilde{S}_{t+1}^{i} \leq \alpha a_{i}\right) I_{1}\left(a_{i}, a_{j}\right)+\mathbb{P}_{t}\left(\tilde{S}^{1}{ }_{t+1} \leq \alpha a_{1}, \tilde{S}^{2} t+1 \leq \alpha a_{2}\right) J_{4} \\
& +\sum_{i, j \in\{1,2\}, i \neq j} \mathbb{P}_{t}\left(\Sigma_{t+1} \leq x, \tilde{S}^{i} t+1 \leq \alpha a_{i}, S_{t+1}^{i} \geq a_{i}-y\right) I_{2}\left(a_{i}, a_{j}\right)+\mathbb{P}_{t}\left(\Sigma_{t+1} \leq x\right) J_{3} \\
& +\int_{0}^{\infty} \int_{0}^{\beta} \mathbb{E}_{t}\left[\mathbb{1}_{X \leq A_{1}, Y \leq A_{2}} p\left(\tilde{r}, \tilde{\theta}, \sigma_{1} \sqrt{0.5}\left(A_{1}-X\right), \sigma_{2} \sqrt{0.5}\left(A_{2}-Y\right)\right)\right]_{s_{i}=S_{t}^{i}} J(\tilde{r}, \tilde{\theta}) d \tilde{r} d \tilde{\theta} .
\end{aligned}
$$

\section{Step 3 - Final calculation}

Remark that $\mathbb{P}_{t}\left(\tilde{S}_{t+1} \leq \alpha a_{1}, \tilde{S}_{t+1}{ }_{t+\alpha} \leq \alpha a_{2}\right)=Q\left(S_{t}^{1}, S_{t}^{2}, 1\right)$ with $Q$ given in Proposition 5 . Also, the conditional law of $\Sigma$, i.e. $\mathbb{P}_{t}\left(\Sigma_{t+1} \leq x\right)$, is given in Lemma 3. Since the calculation method is the same for all the other terms, we only detail here the probability $\mathbb{P}_{t}\left(\Sigma_{t+1} \leq x, \tilde{S}_{t+1}<\alpha a_{1}\right)$. Thus, Markov property gives

$$
\mathbb{P}_{t}\left(\Sigma_{t+1} \leq x, \tilde{S}^{1}{ }_{t+1}<\alpha a_{1}\right)=\mathbb{E}_{t}\left[\mathbb{1}_{S_{t+\frac{1}{2}}^{1}<\alpha a_{1}} \mathbb{P}_{t+\frac{1}{2}}\left(S_{t+\frac{1}{2}}^{1} e^{X_{\frac{1}{2}}^{1}}+S_{t+\frac{1}{2}}^{2} e^{X_{\frac{1}{2}}^{2}} \leq x, \tilde{X}_{\frac{1}{2}}^{1}<\ln \frac{\alpha a_{1}}{S_{t+\frac{1}{2}}^{1}}\right)\right]
$$


where $X_{\frac{1}{2}}^{i}=\frac{\mu_{i}}{2}+\sigma_{i} B_{\frac{1}{2}}^{i}, B^{i}$ two correlated BM independent of $\mathcal{F}_{t+\frac{1}{2}}$. Next, using Theorem 4, we find that $\mathbb{P}_{t+\frac{1}{2}}\left(S_{t+\frac{1}{2}}^{1} e^{X_{\frac{1}{2}}^{1}}+S_{t+\frac{1}{2}}^{2} e^{X_{\frac{1}{2}}^{2}} \leq x, \tilde{X}_{\frac{1}{2}}^{1}<\ln \frac{\alpha a_{1}}{S_{t+\frac{1}{2}}^{1}}\right)$ equals

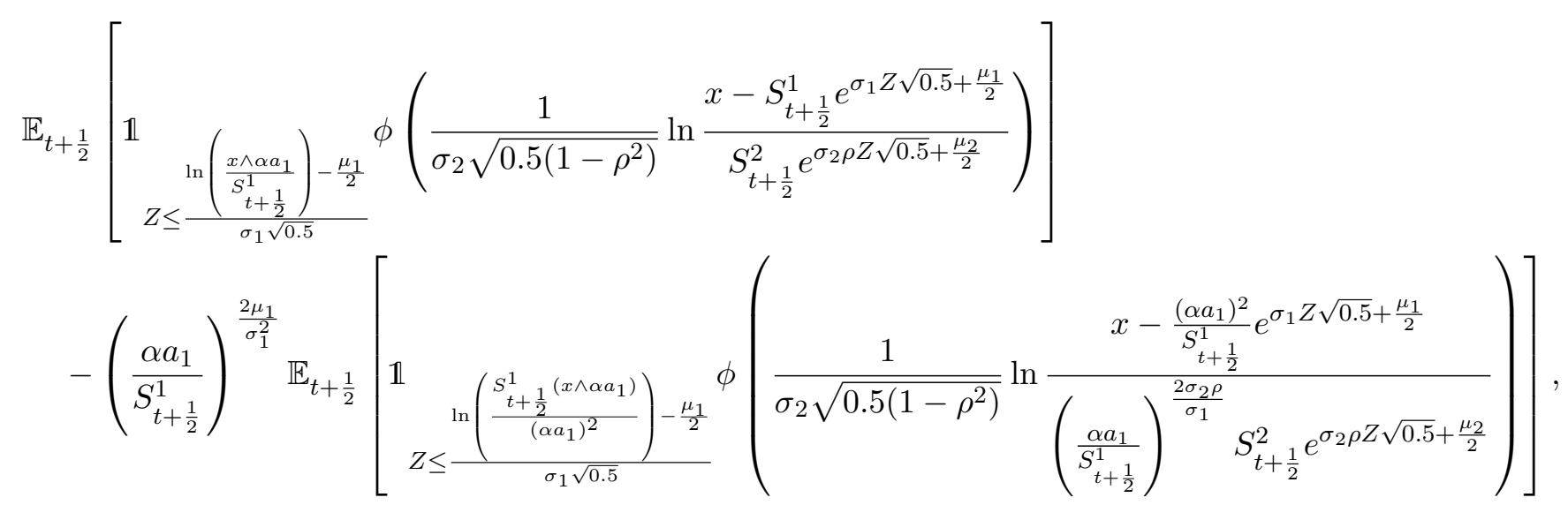

where $Z$ is a standard Gaussian r.v. independent of $\mathcal{F}_{t+\frac{1}{2}}$.

We conclude writing $S_{t+\frac{1}{2}}^{1}=S_{t}^{1}+\frac{\mu_{1}}{2}+\sigma_{1} \sqrt{0.5} X$ (resp. $S_{t+\frac{1}{2}}^{2}=S_{t}^{2}+\frac{\mu_{2}}{2}+\sigma_{2} \sqrt{0.5} Y$ ) with $X, Y$ two correlated standard Gaussian r.v's, that :

$$
\begin{aligned}
& \mathbb{P}_{t}\left(\Sigma_{t+1} \leq x, \tilde{S}^{1}{ }_{t+1}<\alpha a_{1}\right)=\mathbb{E}\left[\mathbb{1}_{X \leq A_{1}, Z \leq \frac{\ln \frac{x \wedge\left(\alpha a_{1}\right)}{s_{1}}-\mu_{1}}{\sigma_{1} \sqrt{0.5}}-X} \phi_{12}\left(s_{1}, s_{2}, X, Y, Z\right)\right]_{s_{1,2}=S_{t}^{1,2}} \\
& -\left(\frac{\alpha a_{1}}{S_{t}^{1}}\right)^{\frac{2 \mu_{1}}{\sigma_{1}^{2}}} e^{-\frac{\mu_{1}^{2}}{\sigma_{1}^{2}}} \mathbb{E}\left[\mathbb{1}_{X \leq A_{1}, Z \leq \frac{\ln \frac{s_{1}\left(x \wedge \alpha a_{1}\right)}{\left(\alpha a_{1}\right)^{2}}}{\sigma_{1} \sqrt{0.5}}+X} \tilde{\phi}_{12}\left(s_{1}, s_{2}, X, Y, Z\right)\right]_{s_{1,2}=S_{t}^{1,2}} .
\end{aligned}
$$

We also find

$$
\begin{aligned}
& \mathbb{P}_{t}\left(\Sigma_{t+1} \leq x, \tilde{S}^{1} t+1 \leq \alpha a_{i}, S_{t+1}^{1} \geq a_{1}-y\right)=\mathbb{E}\left[\begin{array}{l}
\mathbb{1} \ln _{X \leq A_{1}, \frac{a_{1}-y}{s_{1}}-\mu_{1}}^{\sigma_{1} \sqrt{0.5}}-X \leq Z \leq \frac{\ln \frac{x \wedge\left(\alpha a_{1}\right)}{s_{1}}-\mu_{1}}{\sigma_{1} \sqrt{0.5}}-X \\
\phi_{12}\left(s_{1}, s_{2}, X, Y, Z\right)
\end{array}\right]_{s_{1,2}=S_{t}^{1,2}}
\end{aligned}
$$

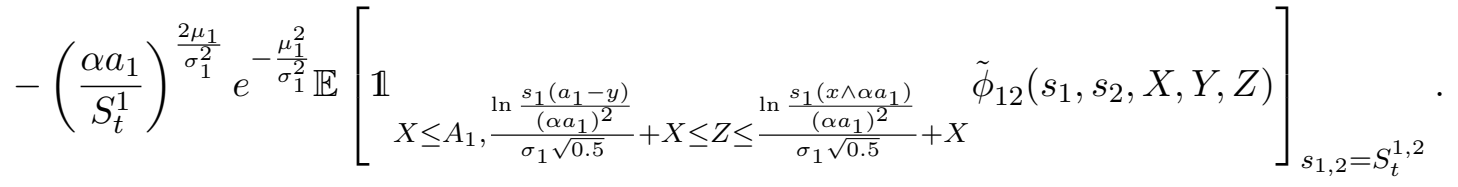

Remark that on $I_{1}, x>\alpha a_{1}$, so :

$$
\begin{aligned}
& \mathbb{P}_{t}\left(\Sigma_{t+1} \leq x, \tilde{S}_{t+1}^{1}<\alpha a_{1}\right)+\mathbb{P}_{t}\left(\Sigma_{t+1} \leq x, \tilde{S}_{t+1} \leq \alpha a_{i}, S_{t+1}^{1} \geq a_{1}-y\right) \\
& =\mathbb{E}\left[J_{1}\left(s_{1}, s_{2}, X, Z\right) \phi_{12}\left(s_{1}, s_{2}, X, Y, Z\right)\right]-\left(\frac{\alpha a_{1}}{S_{t}^{1}}\right)^{\frac{2 \mu_{1}}{\sigma_{1}^{2}}} e^{-\frac{\mu_{1}^{2}}{\sigma_{1}^{2}}} \mathbb{E}\left[J_{2}\left(s_{1}, s_{2}, X, Z\right) \tilde{\phi}_{12}\left(s_{1}, s_{2}, X, Y, Z\right)\right] .
\end{aligned}
$$

This completes the proof. 


\section{Additional Figures}
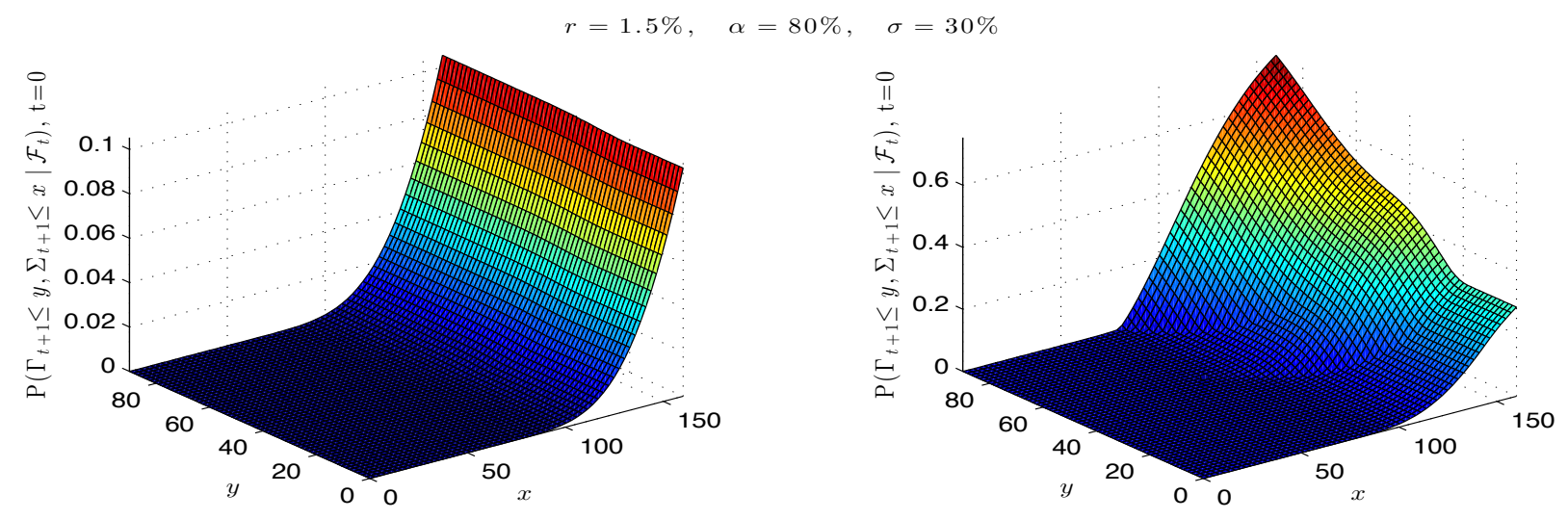

Figure 4: Joint distribution function. The joint probability $\mathbb{P}_{t}\left(\Gamma_{t+1}<y, \Sigma_{t+1} \leq x\right)$ in Theorem 1 (left) and Theorem 2 (right), for $t=0$ with $n=2$. The input parameters are as follows $\alpha=80 \%, r=1 \%$. Comonotonic shares: acquisition values $S_{t_{1}}^{1}=80$ and $S_{t_{2}}^{1}=90$ and initial value $S_{0}^{1}=120$.
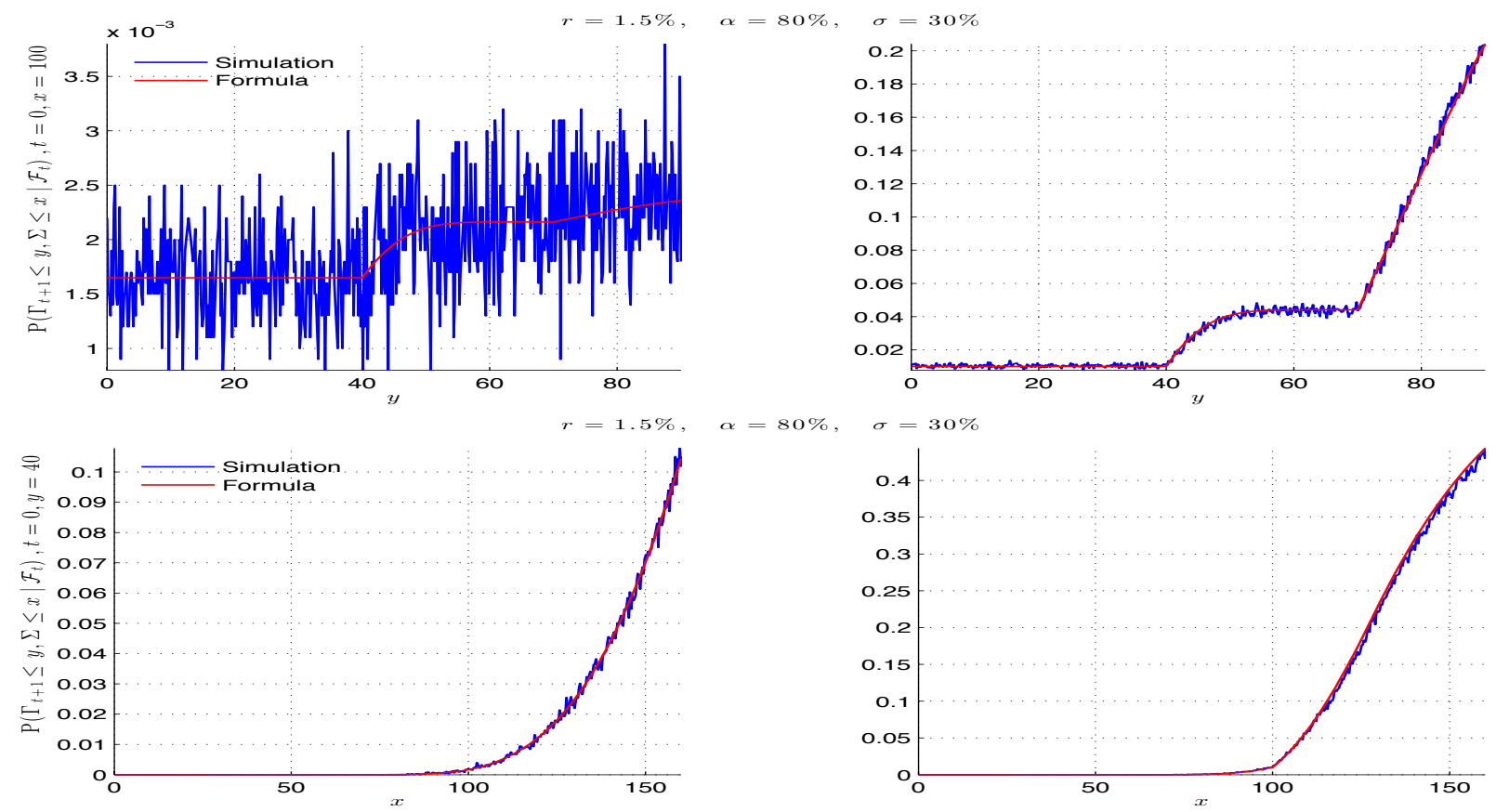

Figure 5: Joint distribution function. The joint probability $\mathbb{P}_{t}\left(\Gamma_{t+1}<y, \Sigma_{t+1} \leq x\right)$ in Theorem 1, for $t=0$, with acquisition values $a_{1}=80$ and $a_{2}=90$ and initial value $S_{0}=120$ (left) and $S_{0}=60$ (right). 